\title{
Die Entwicklung und Morphologie des Skleral= knorpels bei den Anuren.
}

\author{
Von
}

\section{Kenji Yano.}

Aus dem Anatomischen Institut der Keio Universität, Tokyo.

Mit 1 Textfigur und 10 Figuren auf Tafeln XXIV-XXV.

\section{Inhaltsverzeichnis.}

I. Einleitung. . . . . . . . . . . . . . 169

II. Material und Methode. . . . . . . . . . . . . 170

III. Eigene Befunde. . . . . . . . . . . . . . . . 170

A. Bufo vulgaris japonicus (Schlegel). . . . . . 170

Stadienbeschreibung. . . . . . . . . . 171

Zusammenfassung. ............... 178

B. Hyla arborea japonica (Günth er). . . . . . . . 179

Stadienbeschreibung. . . . . . . . . . . 179

Zusammenfassung. . . . . . . . . . . . 181

C. Rhacophorus schlegelii (Günther). . . . . . . 181

Stadienbeschreibung. . . . . . . . . . 181

Zusammenfassung. . . . . . . . . . . 185

D. Polypedates buergeri (Schlegel). ........186

Stadienbeschreibung. . . . . . . . . 186

Zusammenfassung. . . . . . . . . . . . 187

E. Rana japonica (Günther). ......... . 187

Stadienbeschreibung. . . . . . . . . . 187

Zusammenfassung. . . . . . . . . . . 189

IV. Vergleichung und Zusammenfassung. . . . . . . . . . . 190

V. Ergebnisse. . . . . . . . . . . . . . 197

Literaturverzeichnis. . . . . . . . . . . . . . . 199

Erklärung der Abbildungen. . . . . . . . . 200

\section{Einleitung.}

Bezüglich der in der Sklera von verschiedenen Wirbeltieren vorkommenden knorpeligen bzw. knöchernen Elemente ist seit langem von vielen Autoren histologisch wie morphologisch vielfach geforscht worden. So ist auch bei den Anuren die Frage von einer Reihe von Forschern wie 
Bronn('78), Carrière ('85), Helfreich('70), Gaupp ('04) u.s.w. ziemlich genau berücksichtigt worden. Jedoch können wir damit die morphologischen Verhältnisse des Skleralknorpels noch nicht endgültig aufgeklärt finden. Auch über den Entwickelungsmodus des letzteren bei den Anuren finden sich bis jetzt keine Mitteilungen. Ferner herrschen über die Herkunft des Skleralknorpels heute noch zweierlei ganz entgegengesetzte Ansichten; einige Autoren leiten ihn aus dem Primordialkranium her, während die anderen seine sekundäre Natur betonen. Um einen Teil dieser Fragen zu lösen, habe ich in der vorliegenden Untersuchung die Augen von in Japan einheimischen Anuren verfolgt.

\section{Material und Methode.}

Zu den von mir untersuchten Anuren gehören folgende Arten:
A. Bufo vulgaris japonicus (Schlegel),
B. Hyla arborea japonica (Günther),
C. Rhacophorus schlegelii (Günther),
D. Polypedates buergeri (Schlegel),
E. Rana japonica (G ünther).

Zur Fixierung wurde 10\% Formolalkohol angewendet. Die Entkalkung der ausgewachsenen Exemplare nahm ich mit 3\%igem Salpetersäurealkohol vor. Eingebettet wurden der Kopf oder nur das Auge der Tiere in Zelloidin. Die Serienschnitte wurden in senkrechter und horizontaler Richtung zur Körperachse geschnitten. Die Schnittdicke bewegte sich zwischen $20 \mu$ und $50 \mu$.

Als Färbemethode wurden die Stückfärbung durch Eisenhämatoxylin nach Weigert und die Schnittfärbung durch Hämatoxylin(Hansen)Eosin benutzt.

Aus fünf Augen von Bufo vulgaris japonicus und aus zwei Augen von Rhacophorus schlegelii habe ich Wachsplattenmodelle angefertigt. Sie sind zwanzig bis sechs und sechzig mal vergrössert modelliert.

\section{Eigene Befunde.}

\section{A. Bufo vulgaris japonicus (Schlegel).}

Von diesem habe ich acht Augen von vier Larven und zwölf Augen von sieben ausgewachsenen Tieren untersucht. 
Die Entwicklung und Morphologie des Skleralknorpels bei den Anuren.

Stadienbeschreibung.

1. Stulium. Larve ron $29 \mathrm{~mm}$ ganzer Lünge, mit 2 vorleren Extremitäten.

2. Stadium. Larve von $33 \mathrm{~mm}$ ganzer Länge, mit 2 vorderen Extremitïten.

3. Stadium. Larve von $25 \mathrm{~mm}$ ganzer Länge, mit 4 Extremitäten.

t. Stadium. Larve von $13 \mathrm{~mm}$ ganzer Länge, mit 4 Extremitäten.

5. Stadium. Junges Exemplar von $11 \mathrm{~mm}$ Stammlänge (gleich nach der Metamor phose).

6. Stadium. Junges Exemplar von $13 \mathrm{~mm}$ Stammlänge.

7. Stadium. Junges Exemplar von $16 \mathrm{~mm}$ Stammlänge.

8. Stadium. Junges Exemplar von 27 mm Stammlänge.

9. Stadium. Junges Exemplar von $31 \mathrm{~mm}$ Stammlänge.

10. Stadium. Ausgewachsenes Exemplar von $57 \mathrm{~mm}$ Stammlänge.

11. Stadium. Ausgewachsenes Exemplar von $102 \mathrm{~mm}$ Stammlänge.

1. Stadium.

Jarve von $29 \mathrm{~mm}$ ganzer Körperlänge, Stammlänge $10 \mathrm{~mm}$, Schwanzlänge $19 \mathrm{~mm}$ : Die beiden hinteren, etwa $1.5 \mathrm{~mm}$ langen Extremitäten sind noch nicht gut gegliedert.

In diesem Stadium treffen wir schon das ziemlich gut entwickelte Chondrokranium, dessen hintere Partien besonders gut ausgebildet sind; die Ohrkapsel ist fast vollstïndig verknorpelt. Man findet die fast kontinuierliche knorpelige Schädelseitenwand, die jedoch noch in sehr schwacher Entwickelungsstufe steht; darauf befindet sich das gut ausgebildete Foramen opticum. Der Bulbus erweist sich noch nicht als kuglig, sondern dank der noch verhältnismässig planen Hornhaut als becherförmig. Das faserige Skleralgewebe und die Augenmuskeln sind hier ziemlich gut entwickelt; aber ich konnte in der Sklera nirgends eine Knorpelablagerung sehen.

2. Stadium.

Die Larve von $33 \mathrm{~mm}$ ganzer Länge, Stammlänge $11 \mathrm{~mm}$, Schwanzlänge $11 \mathrm{~mm}$ : Die beiden hinteren, $6 \mathrm{~mm}$ langen Extremitäten sind in Ober-, Unterschenkel und Zehen gegliedert. Die linke vordere Extremität ist nur als eine Sprossung erkennbar. Hier erscheint das Chondrokranium etwas besser ausgebildet $\mathrm{zu}$ sein als beim vorigen Stadium. Die Massenzunahme der Augenmuskeln, sowie deren Ur- 
sprung und Ansatz sind viel deutlicher geworden. Die beiderseitigen Augenlider sind noch nicht angedeutet. Man kann hier noch keine Spur vom Skleralknorpel wahrnehmen.

3. Stadium.

Die Kaulquappe hat eine ganze Lünge von $25 \mathrm{~mm}$, die Schwanzlïnge $15 \mathrm{~mm}$; Kopf und Rumpf sind etwas abgeplattet Die vorderen und hinteren Extremitïten sind gut ausgebildet. Das Chondrokranium zeigt jetzt eine bessere Ausbildung als beim vorhergehenden Exemplare. Besonder's in der Orbitalregion hat die Entwickelung ihres häutigen Bodens und der knorpeligen Seitenwand der Schädelhöhle einen bedeutenden Fortschritt gemacht. Die letztere erstreckt sich fast in ganzer Höhe der Gehirnhöhle, vorn von der Ethomoidalregion hinten bis zum vorderen Ohrkapselteil. Das obere Augenlid ist noch nicht angedeutet, aber das untere lässt sich als eine kurze Falte erkennen. An den beiderseitigen Augïpfeln konnte ich noch keine Spur des Skleralknorpels konstatieren.

\section{Stadium.}

Die ganze Lünge dieses Tieres beträgt $13 \mathrm{~mm}$, der Stummelschwanz $4 \mathrm{~mm}$; vier Extremitäten. Im Vergleich mit dem vorigen Exemplare weist das Chondrokranium keine bemerkenswerten Veränderungen auf. Auch hier treffen wir keine Spur von oberer Lidfalte, aber die untere scheint viel deutlicher als rorher ausgebildet zu sein. Auch in den beiderseitigen Augäpfeln ist die Knorpelbildung noch nicht zu finden, die Sklera ist rollständig bindegewebig aufgebaut.

\section{Stadium.}

Das Tier gleich nach der Metamorphose von $11 \mathrm{~mm}$ Stammlänge. Die oberen und unteren Augenlider sind hier bereits gut angedeutet.

Erst bei diesem Stadium kommt beiderseits eine sehr schwache Knorpelbildung in der Sklera zum Vorschein. So kann man auf Schnittpräparaten das Knorpelgewebe als aus einschichtigen Zellen bestehende kleine Stäbchen vorfinden (Taf. XXV, Fig. 6), die meist in dem temporoventralen Teil des Augenhintergrundes, und zwar in der: Stelle zwischen der Insertionszone der geraden Augenmuskeln und Papilla nervi optici liegen. Sie lassen sich alle durch etwa 10 Schnitte $(300 \mu$ weit) der Serie hindurch verfolgen und richten ihre konkave Fläche nach dem Augeninneren zu.

Ich habe aus dem rechten Auge ein 66 mal vergrössertes Wachsplattenmodell angefertigt (Taf. XXIT, Fig. 1), wobei der Bulbus sich nicht als kuglig erweist, sondern ellipsoidisch ist, sodass sein nasotemporaler 
Durchmesser etras lïnger als der dorsoventrale ist. Der Skleralknorpel befimlet sich in der temporoventralen Wand des Augapfels und besteht aus mehreren Plïttchen und Stäbchen von verschiedener Form und Grösse. Aus einer grossen Kuorpelplatte, die gerade ventral von der Eintrittstelle des Nervus opticus sich befindet, gehen schmale Fortsätze von verschiedener Länge aus, von denen die einen frei endigen, während dio :nderen sich untereinander verbindend unregelmässige kleine Ringe oder Notze bilden; so entstehen hier viele inselartige Defekte in der Knorpelsubstanz. Zwei Knorpelplättchen, welche in der Nïhe der Papilla nervi optici eingelagert sind, verbinden sich miteinander durch eine schmale Knorpelbrïcke, die die Papilla nervi optici von rentral halbmondförmig umgibt. Der kürzeste Abstand zwischen der Papilla nervi optici und dieser Knorpelbrücke misst ca. $0.03 \mathrm{~mm}$. Auch findet man zwischen den oben erwähnten grossen Knorpelplättchen mehrere frei isolierte, inselartige Knorpelstäbchen, die einen grössten Durchmesser von $0.04 \mathrm{~mm}$ bis $0.08 \mathrm{~mm}$ haben. Ausserdem befindet sich im nasoventralen Teil des Augenhintergrundes, von der Eintrittstelle des Opticus ca. $1.0 \mathrm{~mm}$ entfernt, ein Knorpelstïckchen, das ca. $0.12 \mathrm{~mm}$ lang und $0.04 \mathrm{~mm}$ breit ist. Daraus kann man sagen, dass der Skleralknorpel in diesem Stadium erst in der ventrotemporalen Wand des Bulbus entsteht.

\section{Stadium.}

Junges metamorphosiertes Exemplar von $13 \mathrm{~mm}$ Stammlänge.

Im Vergleich mit vorigem Stadium ist die Knorpelbildung hier beiderseits verhältnismässig stärker und finden sich auch noch in der Dorsalwand des Bulbus mehrere knorpelige Elemente. Die Grösse der Knorpelplättchen und -spangen sind je nach den Stellen verschieden. Aber in der Nähe der Papilla nervi optici und der Ansatzzone der Augenmuskeln sind sie meistens am dicksten. Dabei scheint auf Schnittpräparaten der Skleralknorpel von der Umgebung vorzüglich scharf abgesetzt zu sein. Ich habe auch aus dem rechten Auge dieses Tieres ein 50 mal vergrössertes Wachsplattenmodell angefertigt (Taf. XXIV, Fig. 2).

Der Bulbus hat hier auch die Form einer Ellipse; der dorsoventrale Durchmesser betrïgt ca. $1.76 \mathrm{~mm}$, der nasotemporale $1.86 \mathrm{~mm}$. Gerade ventral von der Eintrittstelle des Opticus befindet sich eine gut ausgebildete Knorpelplatte. Von ihr gehen viele Fortsätze von verschiedener Form und Grösse aus, und von den grösseren Knorpelfortsïtzen gehen wieder viele kleine Fortsätzchen aus; sie bilden miteinander sich verbindend hie und da zerstreute inselartige Knorpeldefekte von ver- 
schiedener Form und Grösse. Die zwei grossen Fortsätze, welche in der Ventralseite des Bulbus sich befinden, ziehen fast parallel zur $\mathbb{X}$ quatoriallinie des Bulbus nach nasal; deren einer endigt frei, der andere verbindet sich mit dem anderweitigen Knorpclstück. Auch findet man in der Nähe dieser grossen unregelmässigen Knorpelplatte vicle zerstreute, isolierte Knorpelstückchen von verschiedener Form. Selbst das grössto Stïck, das im weitesten Abstand (ca. $1.1 \mathrm{~mm}$ ) vom Opticus sich befindet, überschreitet nicht $0.3 \mathrm{~mm}$ in seinem grössten Durchmesser.

Im Gegensatz zum vorigen Stadium ist der Skleralknorpel auch in der Dorsalwand des Bulbus ausgebildet und besteht aus vier kleinen Knorpelstückchen von unregelmässiger Form und Grösse, die alle proximal von der Ansatzzone der gerarlen Augenmuskeln liegen und nicht bis vorn davon reichen. Das grösste Stück von ihnen hat eine Länge ron ca. $0.3 \mathrm{~mm}$, das kleinste ist kaum $0.02 \mathrm{~mm}$ gross. Der kürzeste Abstand zwischen der Papilla nervi optici und dem Knorpelstück misst ca. $0.6 \mathrm{~mm}$, der weiteste ca. $0.9 \mathrm{~mm}$.

7. Stadium.

Das Tiere hat eine Stammlänge von $16 \mathrm{~mm}$.

Der Skleralknorpel ist viel deutlicher als beim vorhergehenden Stadium in beiden Augen entwickelt und fast symmetrisch. Iusser dem nasalen Teil, wo er als kleine Stücke vorkommt, kann man an der Ventralwand des Bulbus fast immer auf Schnittprïparaten eine sehr gut ausgebildete, lange, bogenfömige Knorpelplatte vorfinden. Diese Knorpelplatte, die mit ihrer Konvexität nach dem Augeninnern gerichtet steht, hat nicht überall gleiche Dicke und Länge. So ist ihre Dicke kurz proximal von der Ansatzzone der Augenmuskeln am stärksten, von hier nimmt sie sowohl proximal- als auch distalwärts allmählich ab und der Knorpel endigt mit spitzigen Rändern. In der nasalen, wie eben erwähnt, und der dorsalen Wand des Bulbus findet man den Knorpel als kleine Stïcke und Plättchen.

Aus dem rechten Auge dieses Tieres habe ich auch ein $50 \mathrm{mal}$ rergrössertes Wachsplattenmodell angefertigt; dieses möchte ich jetzt etwas genauer betrachten (Taf. XXIV, Fig. 3). Zucrst muss besonders hervorgehoben werden, dass erst in diesem Stadium eine Verbindung der ventralen Knorpelplatte mit der dorsalen, auf der temporalen Wand des Bulbus, stattfindet. Verbindet man alle diese Knorpelstücke durch eine Linie, so kann man hier ohne Schwierigkeit eine ringförmige Anordnung des Skleralknorpels um den Opticus sehen, wie es beim ausgewachsenen Hynobius und Onychodactylus der Fall ist. Der ganze Knorpelring hat die Form einer Ellipse, deren längerer Durchmesser nasotemporal, der 
kürzere dagegen dorsoventral steht. Der ganze Umkreis des Ringes beträgt an seinem Distalrand etwa $6 \mathrm{~mm}$ und sein nasotemporaler Durchmesser ca. $2 \mathrm{~mm}$, der dorsoventrale ungefähr $1.7 \mathrm{~mm}$. Abgesehen vom nasodorsalen Teil des Ringes kann man in ihm nirgends eine Unterbrechung finden, wenngleich hie und da die inselartigen Knorpeldefekte sich finden; im nasodorsalen Teil der Knorpelringzone sieht man viele Knorpelstückchen von verschiedener Form und Grösse. Ausserdem finden sich innerhalb des Ringes viele, von diesem ausgehende, unregelmässige Fortsätze und gänzlich isolierte Knorpelstückchen von verschiedener Grösse.

Nun sei das Verhältnis zwischen dem Opticus und Skleralknorpel erwähnt. Der Opticus ist nicht allseitig mit Skleralknorpel umgeben, sondern der letztere weist dorsal eine kurze Unterbrechung auf, worin jedoch wieder ein kleines isoliertes Knorpelstückchen liegt. Daraus kann man sagen, dass das erste Auftreten des Skleralknorpels ventral von der Papilla nervi optici geschieht, dann von hier aus die Fortsätze ausgehen und darauf die Knorpelstückchen dorsal rom Opticus auftreten, um endlich, beide miteinander sich verbindend, einen Ring zu bilden.

8. Stadium.

Das Exemplar hat $27 \mathrm{~mm}$ Stammlänge.

Im Vergleich mit dem vorigen Stadium tragen die beiden Augen hier einen viel besser ausgebildeten Skleralknorpel. Auf Schnittpräparaten kann man ihn als eine unvollkommen geschlossene Knorpelschale erkennen. Abgesehen von 6-7 Schnitten des nasalen Bulbusteiles (180-210 $\mu$ weit) konnte ich immer eine bedeutende Knorpelmasse konstatieren.

Ich habe aus dem linken Auge ein $33 \mathrm{mal}$ vergrössertes Wachsplattenmodell angefertigt (Taf, XXIV, Fig. 4). Als ganzes stellt dieses eine seichte, nicht völlig ausgebildete Knorpelschale dar, die ihre Konvexität nach dem Augeninnern zuwendet. Sie erweist sich nicht als rundlich, sondern als eine Ellipse; deren längerer Durchmesser nasotemporal $(3.2 \mathrm{~mm})$ und der kürzere dorsoventral $(2.8 \mathrm{~mm})$ steht. Ihre Temporalwand hat die grösste Dicke und deren Distalrand bildet eine glatte Ebene im Gegensatz zu denen der anderen Wände, welche durch die grösseren und kleineren Fortsätze ziemlich stark gezackt sind. Die Temporalhälfte des Skleralknorpels zeigt sich schon als eine tief ausgehöhlte, breite Schale, während die andere Hälfte, wegen der mangelhaften Entwickelung, sich nur als eine dünne, wenig konkave Knorpelplatte erweist. Daraus erhellt, dass die Temporalwand in Bezug auf das 
Formverhältnis hier schon seine maximale Entwickelungsstufe erreicht hat und im weiteren Entwicklungsverlauf nur die Dicke und Grösse zunimmt, ohne ihre Gestalt zu verändern. Dann folgt als gut ausgebildete Wand die ventrale, indem man hier nur drei kleine rundliche Knorpeldefekte vorfindet. In der Dorsalwand befinden sich viele unregelmässige Knorpeldefekte von verschiedener Grösse; je weiter nasalwärts wir kommen, desto schwächer wird die Knorpelbildung, indem hier auch viele Defekte sich finden. Aus dem distalen Rand der nasalen Wand gehen viele Knorpelfortsätze distalwärts aus and lassen zwischen sich unregelmässige Einschnitte bilden. Selbst der längste Knorpelfortsatz erreicht nicht den nasalen Pol des Bulbus. Daher ist die Sklera auf dem nasalen Abschnitte der Schnittpräparate gänzlich bindegewebig aufgebaut. Überdies ist die Dicke der Nasalwand beträchtlich dünner als die der anderen. Dieser Befund gibt mir Veranlassung, zu behaupten, dass sich bei den Anuren die Nasalwand des Skleralknorpels im Vergleich mit den anderen Wänden später ausbildet.

Das Foramen opticum cartilaginis sclerae repräsentiert hier noch nicht die rundliche Form, sondern eine unregelmässige. Mit Ausnahme des Dorsalrandes des Foramen, der ziemlich weit entfernt von Papilla nervi optici steht (ca. $0.2 \mathrm{~mm}$ ), liegt der Ventral-, Temporal sowie Nasalrand viel näher dazu. Die Distanz zwischen der Papilla nervi optici und dem Distalrand der Knorpelschale beträgt:

$$
\begin{aligned}
& \text { Dorsal . . . . . . . } 1.3 \mathrm{~mm} \text {, } \\
& \text { Ventral . . . . . . } 1.4 \mathrm{~mm} \text {, } \\
& \text { Nasal . . . . . . . } 2.3 \mathrm{~mm} \text {, } \\
& \text { Temporal . . . . . } 1.4 \mathrm{~mm} \text {. }
\end{aligned}
$$

Daraus erklärt sich, dass der Opticus von dem proximalen Pol der Schale etwas temporalwärts verschoben liegt.

9. Stadium.

Ausgewachsenes Exemplar von $31 \mathrm{~mm}$ Stammlänge.

Die beiderseitigen Augäpfel tragen eine sehr gut ausgebildete Knorpelschale. Abgesehen von wenigen kleinen rundlichen Löchern, die den Blutgefässen zum Durchtritt dienen, kann man in ihr nirgends eine Unterbrechung finden. Ihre Dicke ist eine Strecke weit hinten von der Äquatorzone des Bulbus am stärksten und verdünnt sich allmählich nach proximal, sodass sie am proximalen Bulbuspol eine dünnste Schicht -darstellt. Distalwärts überschreitet der Skleralknorpel die Insertionszone der geraden Augenmuskeln ein wenig und endigt hier mit einem zugeschärften Rand. 
10. Stadium.

Ausgewachsenes Exemplar von $57 \mathrm{~mm}$ Stammlänge.

Von diesem 'Tier habe ich nur das rechte Auge untersucht. Der skleralknorpel verhält sich im grossen und ganzen gleichartig mit dem des vorigen Stadiums, wenngleich er dem ganzen Körperwachstum gemüss an Grösse zugenommen hat. Der Knorpel scheint hier etwas dicker geworden zu sein. In der Mitte der ventralen Bulbuswand reicht der Skleralknorpel distalwärts bis zur Nähe der Ora serrata, wïhrend er in der Dorsalwand schon in der Äquatorzone des Bulbus aufhört.

Aus dieser Serie habe ich ein 20 mal vergrössertes Wachsplattenmodell angefertigt (Taf. XXIV, Fig. 5). Die Knorpelschale erweist sich nicht als rundlich, sondern eiförmig, indem der längere Durchmesser $(6.25 \mathrm{~mm})$ in nasotemporaler und der kürzere $(5.25 \mathrm{~mm})$ in dorsoventraler Richtung steht; die dicke, etwas erweiterte Fläche der Schale ist in der 'lemporalwand des Bulbus und die dünne Spitze in der Nasalwand gelegen.

Abgesehen von dem Foramen opticum und einigen kleinen Löchern, welche den Blutgefüssen zum Durchtritt dienen, zeigt die Knorpelschale nirgends Unterbrechung, sodass sie eime vollständig geschlossene Schale darstellt. Sie ist an der Temporalwand und in der Umgebung des Foramen opticum am dicksten und endigt nach vorn sich allmählich verjüngend mit einem spitzigen Rand. Der Distalrand steht hier nicht in einer, wie geschnittenen Ebene, sondern zeigt je nach den Stellen verschiedene Ebenen. Im allgemeinen scheint die Ventralwand doppelt dicker zu sein als die Dorsalwand. Man findet hier vier kleine Blutgefässlöcher, die vom Foramen opticum etwas entfernt liegen, von denen zwei an der Dorsalwand und zwei andere an der Ventralwand sich befinden. In der Umgebung der Blutgefässlöcher zeigt der Skleralknorpel eine Verdickung. Selbst das dem Foramen opticum nïchst gelegene Gefässloch, das an der Ventralwand sich befindet, liegt vom Foramen etwa $1.25 \mathrm{~mm}$ entfernt. Das Foramen opticum bildet ein rundliches Loch und wird von einem plötzlich zugeschärften Rand begrenzt, doch verdickt er sich davon peripher rasch. Was die Lage dieses Foramen betrifft, so liegt es nicht gerade im proximalen Pol der Knorpelschale, sondern steht etwas nach temporal verschoben, wie die folgende Messung es uns klar zeigt:

Distanz zwischen dem Foramen opticum sclerae und dem Distalrand der Knorpelschale:

$$
\begin{aligned}
& \text { Temporal . . . . . } 3.2 \mathrm{~mm} \text {, } \\
& \text { Nasal . . . . . . . } 4.9 \mathrm{~mm} \text {, } \\
& \text { Dorsal . . . . . . } 3.95 \mathrm{~mm} \text {, } \\
& \text { Ventral . . . . . . } 2.8 \mathrm{~mm} \text {. }
\end{aligned}
$$


11. Stadium.

Ausgewachsenes Exemplar ron $102 \mathrm{nmm}$ Stammlïnge.

Im Vergleich mit dem vorigen Stadium scheint der Skleralknorpel ctwas dünner geworden zu sein im Verhältnis zu seiner vermehrten Grösse; aber sonst findet man keine Besonderheiten.

\section{Yusanmenfassung.}

Beim Bufo vulgaris japonicus lässt sich der Skleralknorpel im Larvenstadium noch nicht konstatieren.

Erst im Stadium 5, Exemplar gleich nach der Metamorphose, tritt er als ein seler schwach ausgebildetes Knorpelstïckchen auf, sodass der Zeitpunkt seines ersten Vorkommens dem des Übergangs rom Wasserzum Landleben sehr nahe steht. Im Stadium 6, Exemplar von $13 \mathrm{~mm}$ Körperlänge, ist der Skleralknorpel etwas stärker ausgebildet und konnte ich auch in der Dorsalwand des Bulbus mehrere Knorpelstücke wahrnehmen. Im Stadium 7, Exemplar von $16 \mathrm{~mm}$ Stammlänge, ist der Skleralknorpel mehr und mehr entwickelt und im Augenhintergrund ringförmig angeorduet. $\Lambda$ ber er erweist sich noch nicht als einen vollstaindigen Knorpelring, sondern zeigt im nasalen Abschnitt viele Unterbrechungen, wo jeloch viele Knorpelstückchen von verschiedener Form und Grösse sich finden. Nun im Staclium 8, $27 \mathrm{~mm}$ langem Exemplar, hat der Skleralknorpel einen bedeutenden Wachstumsfortschritt erfahren. Er repräsentiert hier erst die Form einer Schale, wenngleich in ihm hie und da inselartige Knorpeldefekte von verschiedener Form vorhanden sind. Erst im Stadium 9, $31 \mathrm{~mm}$ langem Exemplar, findet man eine fast vollstïndig ausgebildete Knorpelschale. Lus obigen Befunden kann ich mit Sicherheit einen eigentïmlichen Wachstumsrorgang des Skleralknorpels beim Bufo vulgaris japonicus behaupten, nämlich, dass das Auftreten der knorpeligen Elemente in der Sklera erst gegen das Ende oder gleich nach der Metanorphose beginnt. Die Stelle, wo die Knorpellage zuerst zum Vorschein kommt, ist die ventrotemporale Wand des Augapfels, und zwar hauptsächlich der Ausatzzone der Augenmuskehn entsprechend. Damn tritt die Knorpeleinlagerung auch in der Dorsalund Nasalwand des Bulbus auf. Ciemäss der Entwickelung des Tieres nimmt der Skleralknorpel an (irösse und Dicke zu und stellt einen Knorpelring im Augenhintergrund dar, um endlich die eigentümliche Knorpelschale zu bilden.

Jetzt möchte ich auf den Ausbildungsvorgang beim Foramen opticum sclerae etwas eingehen. Wie schon oben beschrieben, tritt in der 
Ventralseite des Foramen opticum erst im Stadium 5 eine Verknorpelung auf, wohei aber der Knorjel noch nicht bis unterhalb der Eintrittstelle des Opticus reicht, somdern zwischen den beiden ein durchaus bindegewebig aufgebauter skleralteil übrigbleiht. Im stadium 6 reichen die ventral gelagorten Knorjolsogmente bis dicht unterhalb des Opticus und davon gehen die Knorjelfortsit: dorsalwärts aus, die dicht nahe dem Opticus eingelagert werden. Ausserdem befinden sich an der Dorwal- und Temporalseite des Opticus zwei kleine isolierte Ḱnorpelstïckchen. Im Stadium 7, abgesehen ron der Dickenzunahme des Knoryels, sind in der Umgebung des Foramen opticum keine benerkenswerten Veränderungen zu finden. Den völligen C'mschluss de; Foramen mit iler Knorpelsubstanz habe ich erst im Stadium 8, bei $27 \mathrm{~mm}$ langem Eximplar, gretroffen, aber er lïsst sich noch nicht als ein rundliches Loch crkennen, sondern bietet nur dorsal rom Opticus eine unregelmässige Lücke dar. Erst im Statium 9, bei $31 \mathrm{~mm}$ langem Exemplar, trifft man ein vollkommen dargestelltes, rundliches Foramen opticum, das mit einem plötzlich zugeschärften Rand den Opticus dicht umgibt. Daraus kann man sagen, dass auch der knorpelige Umschluss des Foramen opticum sclerae zuerst von ventral beginnt. Von dieser rentralen Knorpelsubstanz gehen die Knorpelfortsätze dorsalwärts entlang der Natiil- und Temporalseite der Eintrittstelle des Opticus aus, die nachher mit dem in der Dorsalseite neugebildeten kleinen Knorpelstückchen sich rereinigen und so ein eigentliches rundes Foramen opticum bilden.

\section{B. Hyla arborea japonica (Gü nther).}

Stadienbeschreibung.

1. Stadium. Larve von $30 \mathrm{~mm}$ Gesamtlänge, hintere Extremitätenanlage erkennbar.

2. Stadium. Larve von $30 \mathrm{~mm}$ Gesamtlänge, mit beiden hintere Extremitäten.

3. Stadium. Larve ron $32 \mathrm{~mm}$ Gesamtlänge, mit 4 Extremitäten.

4. Stadium. Junges Exemplar von $11 \mathrm{~mm}$ Stammlänge, gleich nach der Matamorphose.

5. Stadium. Junges Exemplar von $13 \mathrm{~mm}$ Stammlänge.

6. Stadium. Ausgewachscnes Exemplar von $33 \mathrm{~mm}$ Stammlänge.

1. Stadium.

Die Kaulquappe von $30 \mathrm{~mm}$ Gesamtlänge ; die hinteren Extremitäten sind nur als Inlage erkennbar.

Das Chondrokranium ist schon ziemlich gut ausgebildet und die 
Ohrkapseln sind vollständig verknorpelt. Der Opticus passiert das jetz knorpelig umrandete Foramen opticum, wenngleich die Schädelseitenwand in der mittleren Zone der Orbitalregion noch häutig bleibt.

An den beiderseitigen Augäpfeln kann man noch keine Spur von Knorpeleinlagerung konstatieren.

2. Stadium.

Von diesem Stadium kamen zwei Kaulquappen. von fast derselben Entwicklungsstufe in Betracht. Die ganze Länge beträgt $30 \mathrm{~mm}$, die hinteren Extremitäten sind in Ober- und Lnterschenkel und Zehen gegliedert. Die vorderen Extremitäten sind nur als Anlage erkembar.

Das Chondrokranium zeigt hier keinen bedeutenden Unterschied vom vorigen Stadium. So kann man hier keine Spur von Lidfalten feststellen. Aber im Gegensatz zu fast demselben Stadium des Bufo vulgaris japonicus kann man schon in beiden Bulbi einen ziemlich gut ausgebildeten Skleralknorpel wahrnehmen, der in der ventrotemporalen Wand les Bulbus, und zwar in der Insertionszone der Augenmuskeln liegt (Taf. XXY, Fig. 7). Er stellt ein Knorpelstäbchen und -plättchen von verschiedener Grösse dar, wie es beim Stadium 5 (gleich nach der Metamorphose) von Bufo rulgaris japonicus der Fall ist. Aber in der Dorsal- und Nasalwand des Bulbus konnte ich keine Spur. von Skleralknorpel konstatieren.

3. Stadium.

Larve von $32 \mathrm{~mm}$ Gesamtlänge ; mit allen 4 Extremitäten, die vorderen sind aber noch stummelförmig. Die beiderseitigen Lidfalten sind noch nicht ausgeljildet.

Fast in der Temporalhälfte des Augenhintergrundes kommt der Skleralknorpel nur als einscbichtige Zelleinlagerung vor. Besonders in der Nähe des temporalen Poles des Bulbus erkennt man eine wesentlichc Ausbildung des Knorpels, der hier keine Unterbrechung wabrnehmen lässt. Verfolgt man von temporal nach nasal, so trifft man das Gebiet, wo der Knorpel hie und da als kleine zerstreute Knorpelstückchen sich vorfindet. Er lässt sich bis zur Nähe der Eintrittstelle des Opticus verfolgen. Das Foramen opticum sclerae ist hier noch ganz bindegewebig aufgebaut.

t. Stadium.

Junges Exemplar unmittelbar nach der Metamorphose ; die Stammlänge beträgt $11 \mathrm{~mm}$.

Der Skleralknorpel lässt im Vergleich mit vorhergehendem Stadium wesentliche Veränderungen erkennen. Als ganzes stellt er eine schon ziemlich gut ausgebildete Knorpelschale dar, aber man kann an der 
Nasalwand des Bulbus keine Knorpelsubstanz wahrnehmen; solche knorpelfreie Zone erstreckt sich auf etwa 10 Schnitte $(250 \mu)$. Die Knorpelschale zeigt ausserdem hie und da viele Unterbrechungen von verschiedener Grösse und reicht distalwärts bis vor die Insertionszone der geraden Augenmuskeln. Das Foramen opticum sclerac stellt hier schon ein knorpeliges rundliches Loch dar.

5. Stadium.

Exemplar von $13 \mathrm{~mm}$ Stammlïnge.

Dic beiderseitigen Augäpfel enthalten jetzt eine fast vollkommen ausuebildete Knorpelschale, indem der Knorpel an der nasalen Wand des Bulbus eine bedeutende Fortbildung erfahren hat.

6. Stadium.

Ausgewachsenes Exemplar von $33 \mathrm{~mm}$ Stammlänge.

Im Verhältnis zur Grösse der Knorpelschale ist ihre Dicke gering, sie ist in der Ansatzzone der Augenmuskeln und an der Temporalwand des Bulbus am stärksten, während sie an anderen Teilen als eine dünne einschichtige Knorpeleinlagerung vorkommt. Ausserdem findet man an der dünnen Knorpelzone hie und da viele grosse Knorpelunterbrechungen.

\section{Zusammenfassung.}

Bei Hyla arborea japonica kamn man immer auch einen hyalinen Knorpel in der Sklera wahrnehmen, welcher die Form einer sehr dünnen Schale hat. Das Auftreten der knorpeligen Elemente in der Sklera beginnt in einem rerhältnismässig frühen Larvenstadium, wobei das Chondrokranium ziemlich gut entwickelt ist, und zwar sind die Ohrkapseln vollständig verknorpelt, was für das 2. Stadium (Exemplar von 30 mm Gesamtlänge, die hinteren Extremitäten ganz gut gegliedert und die vorderen nur als Sprossung erkennbar) gilt.

Zuerst tritt der Knorpel an der temporoventralen Wrand des Bulbus entsprechend der Ansatzzone der Augenmuskeln auf. In dem etwas fortgeschrittenen Stadium 3 (32 mm Gesamtlänge, mit 4 Extremitäten) dehnt sich der Knorpel betrïchtlich nach dorsotemporal aus, sodass der temporale Pol des Bulbus von knorpeligen Elementen völlig geschlossen worden ist. Unmittelbar nach der Metamorphose findet man den Knorpel als eine bereits ziemlich gut dargestellte Schale.

\section{Rhacophorus schlegelii (Günther).}

Stadienbeschreibung.

1. Stadium. Larve von $30 \mathrm{~mm}$ Stammlänge, hintere Extremitätenanlage erkennbar. 
2. Stadium. Larve von $32 \mathrm{~mm}$ Stammlänge, hintere Extremitäter stummelförmig.

3. Stadium. Larve von $40 \mathrm{~mm}$ Stammlänge, mit beiden hinteren Extremitüten.

4. Stadium. Larve von $38 \mathrm{~mm}$ Stammlänge, linke vordere Extremitätenanlage erkennbar.

5. Stadium. Larve von $39 \mathrm{~mm}$ Stammlänge, mit 4 Extremitäten.

6. Stadium. Larve von $26 \mathrm{~mm}$ Stammlänge, mit 4 Extremitäten.

7. Stadium. Exemplar gegen das Ende der Metamorphose, Gesantlänge $19 \mathrm{~mm}$, Schwanz stummelförmig.

8. Stadium. Exemplar gleich nach der Metamorphose, $14 \mathrm{~mm}$ Stammlänge.

9. Stadium. Ausgewachsenes Exemplar ron $47 \mathrm{~mm}$ Stammlänge.

1. Stadium.

Die Kaulquappe von $30 \mathrm{~mm}$ Länge; hintere Extremitiiten als eine höckerartige Sprossung erkennbar.

Das (hondrokranium ist schon ziemlich gut ausgebildet; besonders haben die hinteren Schädelpartien eine wesentliche Fortbildung erfahren, die Ohrkapseln sind fast vollständig verknorpelt und hinter diesen lässt sich jetzt die Occipitalregion gut unterscheiclen. Dennoch ist das Foramen opticum nicht gut dargestellt, sodass der Opticus durch die häutige Schïdelseitenwand austritt.

Der Bulbus erweist sich noch nicht als kuglig, sondern als becherförmig, indem er noch eine etwas plane Hornhaut hat. Die Augenmuskeln zeigen hier schon eine gute Ausbildung. In der Nïhe des Temporalpoles und auch in der Gegend des nasalen Drittels des Augenhintergrundes trifft man eine sehr schwache Knorpelbildung in der Sklera, die entsprechend der Ansatzzone der Augenmuskeln liegt (Taf. XXY, Fig. 8). Der Knorpel besteht aus wengen Knorpelzellen und sehr spärlicher Grundsubstanz (Vorknorpel) und hat die Form eines Plättchens, das rom umgebendon jungen faserigen Skleralgewebe nicht so scharf abgesetzt ist. Das Plättchen lässt sich etwa durch 2-3 Schnitte verfolgen und ist 4-5 mal so lang als breit; es hat eine innere konkave Fläche und eine äussere Konvexität zum äusseren dünnen faserigen Skleralgewebe hin. Ausserdem kommen hie und da im jungen faserigen Teil wenige blasige Knorpelzellen vor. So kann man hier die Umwandlung des faserigen Skleralgewebes zur Knorpelsubstanz sehr klar beobachten.

2. Stadium.

Kaulquappe von $32 \mathrm{~mm}$ Länge ; Stammlänge $11 \mathrm{~mm}$, Schwanzlänge 
$21 \mathrm{~mm}$. Die hinteren Extremitïten sind stummelförmig (ca. $2 \mathrm{~mm}$ ) und Oher- und Unterschenkel, Fuss und '/ehen noch nicht gut differenziert.

Das ('hondrokranium zeigt hier keine wesentlichen Verïnderungen im Vergleich mit dem vorhergehenden stalium. Der Op,ticus tritt inmer noch durch die lä̈utige schä̈delseitenwand in den ()rlitalraum alls.

Der Skleralknorpel ist heiderscits viel weiter gediehen als beim vorhergehenden stadium. So kann man hier eine sehr stark ausgebreitete Knorpeleinlagerung wahrnehmen mit Ausnahme des nasalen $\mathrm{Ab}$ schnittes des Bulbus (etwa 10 Schnitte, 200\% breit), wenngleich sie nur aus dünnen einschichtigen Knorpelzellen bestelt.

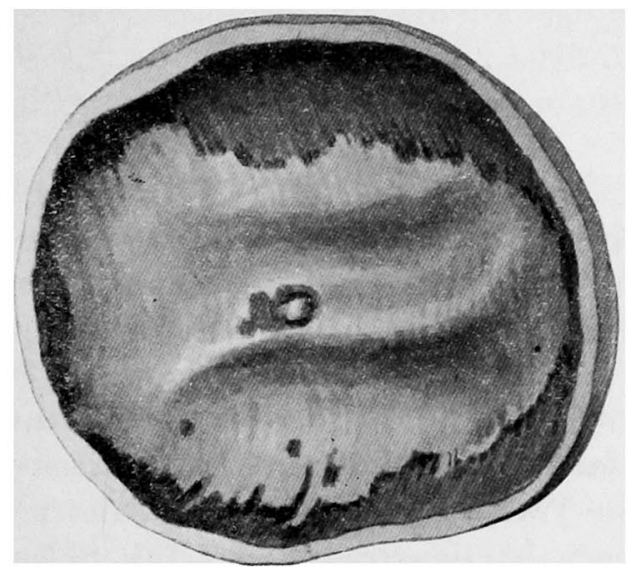

Fig. 1.

Modell des rechten Auges einer $33 \mathrm{~mm}$ langen Kaulquappe von Rhacophorus schlegelii, nur die hintere Augenhälfte dargestellt; 50 fach vergrössert modelliert, (1:1). Die Knorpelsubstanz ist weisslich gezeichnet. Mitten in der Knorpelzone ist die Sehnervenpapille als ein rundlicher Höcker zu sehen. Das Präparat ist durch die Fixierung geschrumpit, wodurch zwei Falten entstanden sind.

Ich habe aus dem rechten 1 uge dieses Tieres ein 50 fach vergrössertes Wachsplattenmodell angefertigt. Der Bulbus erweist sich auch noch nicht als kuglig, sondern zeigt eine etwas abgeplattete ellipsoidische Form ; er ist dorsoventral kürzer, nasotemporal lïnger. Schon in diesem Stadium nimmt der Skleralknorpel den grössten Teil des Augenhintergrundes um die Lintrittstelle des Opticus ein. Er stellt aber noch nicht eine vollständige Schale dar, sondern nur einen dünnen basalen Teil der Schale. Distalwärts erreicht er nicht die Augenäquatorzone, sondern hört schon proximalwärts weit davon entfernt mit einem unregelmässigen Rand auf. Das Foramen opticum ist hier schon ron knorpeliger Sub- 
stanz vollkommen umschlossen worden, wenngleich es noch kein runc liches Loch darstellt.

3. Stadium.

Kaulquappe von $40 \mathrm{~mm}$ Gesamtlänge, Schwanzlänge $28 \mathrm{~mm}$. Die äussere Form der ca. $8 \mathrm{~mm}$ langen hinteren Extremitäten ist fast vollständig herausmodelliert.

Das Chondrokranium zeigt hier keine wesentlichen Veränderungen wie vorher. Der viel deutlicher als beim vorhergehenden Stadium entwickelte Skleralknorpel kommt in beiden Augen vor.

Ich habe auch aus dem rechten Auge dieses Tieres ein fünfzigmal vergrössertes Wachsplatenmodell angefertigt. Als ganzes zeigt sich der Skleralknorpel als eine seichte Knorpelschale. In der Temporal- und Ventralwand des Bulbus reicht sein Distalrand bis zur Augenäquatorzone. Das Foramen opticum hat hier keine bedeutenden Veränderungen erfahren.

4. Stadium.

Die Kaulquappe $38 \mathrm{~mm}$ lang, Stammlänge $8 \mathrm{~mm}$, Schwanzlänge $30 \mathrm{~mm}$; die beiden hinteren Extremitäten sind ca. $18 \mathrm{~mm}$ lang, die linke vordere Extremität ist nur als Anlage erkennbar. Der Körper ist etwas abgeplattet.

In der kontinuierlich knorpeligen Schädelseitenwand findet sich jetzt ein sehr grosses Foramen opticum. Der Opticus geht durch dessen untere Hälfte dem Trabekel dicht anliegend, und der übrige grössere Foramenteil ist noch häutig geschlossen. Der Skleralknorpel verhält sich beiderseits im grossen und ganzen fast gleich wie der im vorigen Stadium.

5. Stadium.

Die Kaulquappe $39 \mathrm{~mm}$ lang, Stammlänge $14 \mathrm{~mm}$, Schwanzlänge $25 \mathrm{~mm}$; mit 4 Extremitäten.

In diesem Stadium ist das Wrachstum des Chondrokraniums bedeutend fortgeschritten. Die beiderseitigen Bulbi enthalten jetzt fast vollkommen ausgebildete Knorpelschalen, deren Dicke beträchtlich zugenommen hat. Im Vergleich mit dem vorhergehenden Stadium zeigt sich in der Dorsalwand des Bulbus viel bedeutendere Ausbildung des Knorpels in Dicke und Ausbreitung. Distalwärts reicht der Knorpel bis vor die Ansatzzone der geraden Augenmuskeln. In der Mitte der Dorsalwand der Knorpelschale, entsprechend der Ansatzzone der Augenmuskeln liegen einige rundliche Löcher, die den Gefässen zum Durchtritt dienen. Das Foramen opticum erscheint rundlich. Die Knorpelschale ist an der Temporalwand des Bulbus und in der Nähe des 
oramen am dicksten, verdünnt sich distalwärts allmählich und endigt lit spitzigem Rand.

6. Stadium.

Excmplar gegen Ende der Metamorphose, Gesamtlünge $19 \mathrm{~mm}$, tammlänge $13 \mathrm{~mm}$, Schwanzlinge nur $6 \mathrm{~mm}$, schon beträchtlich eduziert.

Im Vergleich mit dem vorigen Stadium verhält sich der Skleralknorel beiderseits ganz gleich.

\section{Stadium.}

Junges Exemplar von $14 \mathrm{~mm}$ Stammlänge gleich nach der Metamorhose. In diesem Stadium ist der Skleralknorpel in Dicke und Grösse ıur sehr wenig zugenommen. Sonst rerhält er sich völlig übereinstimnend mit dem Stadium 5 und 6 .

8. Stadium.

Ausgewalchsenes Exemplar von $47 \mathrm{~mm}$ Stammlänge.

Von diesem habe ich nur das rechte Auge untersucht. Im Verhältnis zur Grösse scheint die Dicke der Knorpelschale viel schwächer zu sein. Distalwärts reicht der Knorpel etwas nach vorn über die Insertionszone der geraden Augenmuskeln hinaus, erreicht aber nie den Sklerokornealrand. In der Mitte der Dorsal- und Ventralwand der Knorpelschale, entsprechend der Insertionszone der Muskeln, finden sich einige kleine rundliche Gefässlöcher.

\section{Zusammenfassung.}

Beim Rhacophorus schlegelii (Günther) kommt der Skleralknorpel schon im Stadium 1 (30 mm Gesamtlänge) vor, wobei das Chondrokranium schon ziemlich gut ausgebildet ist, und zwar die Ohrkapseln fast vollständig verknorpelt sind. Hier besteht der Knorpel nur als eine Lage von rundlichen Zellen, wie sie der Vorknorpel besitzt; die Grundsubstanz ist nur in Spur nachweisbar. Ein solcher Vorknorpel liegt an der Temporalseite und dem nasalen Drittel des Bulbus, entsprechend der Ansatzzone der Augenmuskeln. Beim Stadium 2 (32 mm Gesamtlänge, beide hintere Extremitäten stummelförmig) ist der Knorpel plötzlich stark entwickelt und schon im grössten Teil des Augenhintergrundes ausgebreitet. Im Stadium 3 (40 mm Gesamtlänge) ist der Knorpel in Temporal- und Ventralwand des Bulbus viel besser ausgebildet und reicht schon bis zur Augenäquatorzone. Im Stadium 5 (39 mm Gesamtlänge, mit 4 Extremitäten), wo das Chondrokranium bedeutenden Fortschritt gemacht hat, ist der Knorpel plötzlich stark entwickelt, sodass man hier zum erstenmal eine fast vollkommen ausgebildete Knorpelschale sieht, 
die distalwärts über die Ansatzzone der geraden Augenmuskeln hin reicht, aber nirgends den Hornhautrand erreicht.

\section{Polypedates buergeri (Schlegel).}

Stadienbeschreibung.

1. Stadium. Larve von $25 \mathrm{~mm}$ Gesamtlänge.

2. Stadium. Larve von $33 \mathrm{~mm}$ Gesamtlänge, mit beiden hinterer Extremitäten.

3. Stadium. Larve von $26 \mathrm{~mm}$ Gesamtlänge, mit 4 Extremitäten.

4. Stadium. Larve von $18 \mathrm{~mm}$ Gesamtlänge, mit 4 Extremitäten.

5. Stadium. Junges Exemplar von $14 \mathrm{~mm}$ Stammlänge gleicl nach der Metamorphose.

6. Stadium. Zwei ausgewachsene Exemplar von 32 und $35 \mathrm{~mm}$ Staminlänge.

1. Stadium.

Kaulquappe von $25 \mathrm{~mm}$ Länge; Stammlänge $10 \mathrm{~mm}$, Schwanzlänge $15 \mathrm{~mm}$. Die beiden hinteren Extremitäten sind nur als Sprossung erkennbar.

Das Chondrokranium ist ziemlich gut ausgebildet und zwar sind die Ohrkapseln schon vollständig verknorpelt. Dennoch zeigt die Schädelseitenwand noch keine genügende Ausbildung, sodass der Opticus durch die häutige Wand aus dem Cavum cranii austritt, dem Trabekel dicht anliegend.

An den beiderseitigen Augäpfeln konnte ich keine Spur ron Knorpeleinlagerung konstatieren.

2. Stadium.

Kaulquappe ron $33 \mathrm{~mm}$ Gesamtlänge, Stammlänge $12 \mathrm{~mm}$, Schwanzlänge $21 \mathrm{~mm}$. Die hinteren Extremitäten sind deutlich ausgegliedert, die vorderen nur als höckerige Sprossung erkennbar.

Im Vergleich mit dem vorigen Stadium zeigt das Chondrokranium eine wesentliche Fortbildung, man findet hier an der dicken kontinuierlich knorpeligen Schädelseitenwand ein grosses Foramen für den Opticus,

An den beiderseitigen Augen kann man eine bedeutende Ausbildung der Knorpelsubstanz wahrnehmen (Taf. XXV, Fig. 9). Wenngleich der Skleralknorpel nur als einschichtige Zellenlage auftritt, so verbreitet er sich doch im grösseren Teil des Augenhintergrundes. So kann man auf fast allen Schnittpräparaten beiderseits einen kontinuierlichen sichelformigen Knorpel wahrnehmen, mit Ausnahme der kleinen Strecke der Nasalwand des Bulbus (6-9 Schnitte). Das Foramen opticum ist von 
knorpeligen Elementen vollständig umgeben. Distalwärts reicht der Knorpel jedoch nicht bis zur Augenäquatorzone, sondern hört schon etwas proximal davon auf.

3. Stadium.

Kaulquappe von $26 \mathrm{~mm}$ Gesamtlänge, Stammlänge $10 \mathrm{~mm}$, Schwanzlänge $16 \mathrm{~mm}$; mit 4 Extremitäten.

Im Vergleich mit dem vorhergehenden Stadium findet man hier eine wesentliche Ausbildung des Knorpels in nasaler Wand des Bulbus, sodass man die Knorpelsubstanz durch alle Schnitte konstatieren kann.

4. Stadium.

Kaulquappe von $18 \mathrm{~mm}$ Gesamtlänge; Stammlänge $14 \mathrm{~mm}$, Schwanzlïnge nur $4 \mathrm{~mm}$, also schon beträchtlich reduziert.

Der Skleralknorpel weist keine Veränderungen abgesehen von der Ventralwand des Bulbus auf. Infolge deren distaler Verbreitung bis zur Augenäquatorzone hat er jedoch an Tiefe zugenommen und eine typische Schalenform bekommen.

5. Stadium.

Junges Exemplar von etwa $14 \mathrm{~mm}$ Stammlänge gleich nach der Metamorphose.

Die beiderseitigen Augen enthalten eine sehr gut ausgebildete vollständige Knorpelschale, die in Dicke etwas stärker geworden zu sein scheint als beim vorigen Stadium. Übrigens zeigen sich keine wesentlichen Veränderungen.

6. Stadium.

Zwei ausgewachsene Exemplare von 32 und $35 \mathrm{~mm}$ Stammlänge. Der Skleralknorpel hat dem allgemeinen Körperwachstum gemäss an Grösse und Dicke zugenommen. Er trägt die Form einer seichten Schale. Distalwärts erreicht er nicht die Augenäquatorzone. Übrigens verhält er sich ganz ähnlich wie der von anderen ausgewachsenen Anuren.

\section{Zusammenfassung.}

Beim Polypedates buergeri (Schlegel) beginnt die Knorpelbildung in der Sklera verhältnismässig spät im Vergleich mit dem Rhacophorus schlegelii. So konnte ich in Stadium 1 (25 mm Gesamtlänge) keine Knorpeleinlagerung in der Sklera wahrnehmen. Beim Stadium 2 (33 mm Gesammtlänge) kommt er im grössten Teil des Augenhintergrundes vor. Und im Stadium 4, gegen das Ende der Metamorphose, ist der Skleralknorpel viel stärker ausgebildet und weist eine eigentürnliche Knorpelschale auf. 


\section{E. Rana japonica (Günther).}

Stadienbeschreibung.

1. Stadium. Larve von $32 \mathrm{~mm}$ Gesamtlänge.

2. Stadium. Larve von $33 \mathrm{~mm}$ Gesamtlänge, mit beiden hinteren Extremitäten.

3. Stadium. Larve von $36 \mathrm{~mm}$ Gesamtlänge, mit beiden hinteren Extremitäten.

4. Stadium. Larve von $40 \mathrm{~mm}$ Gesamtlänge, mit beiden hinteren Extremitäten.

5. Stadium. Larve von $38 \mathrm{~mm}$ Gesamtlänge, mit 4 Extremitäten.

6. Stadium. Larve von $32 \mathrm{~mm}$ Gesamtlänge, mit 4 Extremitäten.

7. Stadium. Larve von $27 \mathrm{~mm}$ Gesamtlänge, mit 4 Extremitäten.

8. Stadium. Larve von $24 \mathrm{~mm}$ Gesamtlänge, mit 4 Extremitäten.

9. Stadium. Exemplar von $20 \mathrm{~mm}$ Gesamtlänge gegen das Ende der Metamorphose.

10. Stadium. Ausgewachsenes Exemplar von $32 \mathrm{~mm}$ Stammlänge.

1. Stadium.

Die Kaulquappe von $32 \mathrm{~mm}$ Gesamtlänge; Stammlänge $11 \mathrm{~mm}$, Schwanzlänge $21 \mathrm{~mm}$ : Die beiden hinteren Extremitäten sind nur als Anlage erkenmbar.

In diesem Stadium ist das Wachstum des Chondrokraniums schon bedeutend fortgeschritten, und zwar sind die Ohrkapseln fast rollständig verknorpelt. In der kontinuierlich knorpeligen Schädelseitenwand findet sich jetzt ein sehr gut dargestelltes rundliches Foramen opticum.

An den beiderseitigen Augäpfeln kann man noch keine Knorpel- bildung finden.

2. Stadium.

Kaulquappe ron $33 \mathrm{~mm}$ Gesamtlänge, Stammlänge $11 \mathrm{~mm}$, Schwanzlänge $22 \mathrm{~mm}$ : Die beiden hinteren Extremitäten sind stummelförmig und ca. $3 \mathrm{~mm}$ lang.

Ebenso wie im vorhergehenden Stadium ist an den beiderseitigen Augäpfeln noch keine Knorpelbildung aufgetreten ; das Chondrokranium steht auch auf fast gleicher Entwicklungsstufe.

3. Stadium.

Kaulquappe von $36 \mathrm{~mm}$ Gesamtlänge, Stammlänge $13 \mathrm{~mm}$, Schwanzlänge $23 \mathrm{~mm}$. Die hinteren Extremitäten sind ziemlich gut gegliedert und betragen ca. $4 \mathrm{~mm}$.

Erst von diesem Stadium an trifft man beiderseits eine sehr schwache 
Knorpelbildung in der Sklera (Taf. XXV, Fig. 10). Eine kleine Menge knorpeliger Elemente liegt an der temporoventralen Wand des Bulbus und erscheint an der Ansatzzone der Augenmuskeln viel deutlicher ausgebildet zu sein.

\section{Stadium.}

Kaulquappe von $40 \mathrm{~mm}$ Gesamtlänge ; Stammlänge $12 \mathrm{~mm}$, Schwanzlänge $28 \mathrm{~mm}$ : Die hinteren Extremitäten sind in Ober- und Unterschenkel, Fuss und Zehen sehr gut gegliedert Linke vordere Extremität ist nur als Anlage erkennbar.

Abgesehen vom grössten Teil der nasalen und dorsalen Wand des Bulbus, wo der Knorpel als einige kleine Spangen auftritt, kann man überall einen kontinuierlichen Skleralknorpel wahrnehmen. Besonders in dem temporoventralen Teil des Bulbus ist der Knorpel ziemlich dick. Das Foramen opticum wird von der Knorpelsubstanz umschlossen.

5. Stadium.

Die Kaulquappe $38 \mathrm{~mm}$ lang, Stammlänge $10 \mathrm{~mm}$, Schwanzlänge $28 \mathrm{~mm}$; mit 4 Extremitäten, von denen die vorderen stummelförmig sind.

Die beiderseitigen Bulbi enthalten schon eine ziemlich gut ausgebildete Knorpelschale. Während an der Ventralwand des Bulbus der Distalrand der Schale fast bis zur Augenäquatorzone reicht, hört sie an der Dorsalwand eine Strecke weit entfernt proximal vom Äquator auf.

6. Stadium.

- Die Kaulquappe von $32 \mathrm{~mm}$ Gesamtlänge, Stammlänge $11 \mathrm{~mm}$, Schwanzlänge $21 \mathrm{~mm}$, mit 4 Extremitäten.

Der Skleralknorpel verhält sich beiderseits fast gleich wie der des vorigen Stadiums.

7. Stadium.

Die Kaulquappe von $27 \mathrm{~mm}$ Gesamtlänge, Stammlänge $12 \mathrm{~mm}$, Schwanzlänge $15 \mathrm{~mm}$.

Die beiderseitigen Augäpfel tragen auch eine gut ausgebildete Knorpelschale, die hier an Dicke gegen früher etwas zugenommen zu sein scheint.

8. Stadium.

Die Kaulquappe von $24 \mathrm{~mm}$ Gesamtlänge, Stammlänge $11 \mathrm{~mm}$, Schwanzlänge $13 \mathrm{~mm}$, mit 4 Extremitäten.

Hier scheint beiderseits die Knorpelschale viel tiefer geworden zu sein, indem die Dorsalwand der Schale etwas nach distal ausgebreitet ist.

9. Stadium.

Das Exemplar gegen Ende der Metamorphose von $20 \mathrm{~mm}$ Gesamt- 
länge, Stammlänge $16 \mathrm{~mm}$, Schwanzlänge nur $4 \mathrm{~mm}$, beträchtlich reduziert.

Hier ist eine viel deutlicher ausgebildete Knorpelschale als bein vorhergehenden Stadium vorhanden.

10. Stadium.

Ausgewachsenes Exemplar von ca. $32 \mathrm{~mm}$ Stummlänge.

Von diesem habe ich nur das rechte Auge untersucht. In der Mitte des Bulbus erreicht die Dorsal- und Ventralwand der Knorjelschale distalwärts die Augenäquatorzone und endigt hier mit einem spitzigen Rand. An der Dorsal- und Ventralwand der Schale sehr nahe dem Distalrand finden sich einige rundliche Gefässlöcher. Die ventrale Wrand der Schale ist etwa zweifach dicker als die dorsale.

\section{Zusammenfassung.}

Bei Rana japonica (Günther) kommt der Skleralknorpel etwas später vor, als bei Rhacophorus schlegelii und Polypedates buergeri. So konnte ich erst im Stadium 3 (36 mm Gesamtlänge) eine schwache Knorpeleinlagerung in der Sklera, und zwar in der temporoventralen Wand des Bulbus, wahrnehmen. Im Stadium 4 (40 mm Gesamtlïnge) kommt er am grössten Teil des Augenhintergrundes vor, mit Ausnahme der Nasal- und Dorsalwand des Bulbus. Im Stadium 5 (38 mm Gesamtlänge, mit 4 Extremitäten) kann man beiderseits schon eine eigentümliche Knorpelschale vorfinden. Weiter darauf zeigt der skleralknorpel keine bedeutenden Teränderungen in seiner Form, wemngleich er gemüss der allgemeinen Entwicklung der Tiere an Grösse und Dicke zunimmt.

\section{Vergleichung und Zusammenfassung.}

Der Zeitpunkt des ersten Auftretens von Knorpelelementen in der Sklera bei den Anuren ist je nach den Formen des Tieres verschieden. Aber in den meisten Fällen beginnt die Ausbildung des Skleralknorpels in einem rerhältnismässig frühen Larvenstadium, wo auch das Chondrokranium ziemlich gut entwickelt ist, so z. B. die Ohrkapseln schon verknorjelt sind.

Bei Hyla arborea jajonica gilt dies für ein Larvenstadium von $30 \mathrm{~mm}$ Gesamtlänge, wo die beiden hinteren Extremitäten in Fuss, Cnter- und Oberschenkel gegliedert sind. Und unmittelbar nach der Netamorphose kommt der Knorpel bereits als eine ziemlich gut ausgebildete Schale vor. 
Bei Rhacophorus schlegelii kommt der Skleralknórpel auch zuerst an der Larve von $30 \mathrm{~mm}$ Gesamtlänge ror, wo jedoch die hinteren Exremititen nur erst als Anlage erkennbar sind. (iegen das Encle der Metamorphose stellt er auch eine vollkommene Knorpelschale dar.

Beim Polypedates buergeri konnte ich im Larvenstadium ron $33 \mathrm{~mm}$ Gesanntlänge einen sehr gut ausgebildeten Skleralknor\}el im grössten Teil des lugenhintergrundes konstatieren, der auch gegen das Ende der Metamorphose eine eigentümliche Schale darstellt.

Bei Rina japonica tritt der Skleralknorpel erst in der Larve ron $36 \mathrm{~mm}$ Gesamtlänge mit beiden hinteren, ziemlich gut gegliederten Extremitïten auf und gegen das Ende der Metamorphose kommt er bei den beiderseitigen Augen als eine sehr gut ausgebildete Schale vor.

Daraus möchte ich sagen, dass bei obigen vier Formen von Anuren der Skleralknorpel erst im Anfangstadium der Metamorphose zum Vorschein kommt, sich weiter entwickelt und gegen das Ende der Metamorphose eine eigentümliche Schalenform aunimmt; und zwar bewegt sich der Zeitpunkt seines ersten Auftretens von der Zeit, wo die hinteren Extremitïten sich nur als Anlage erkennen lassen, bis zur Zeit, wo die vorderen Extremitüten als Sprossung zu Tage treten.

Im Gegensatz dazu verhält sich die Sache beim Bufo vulgaris japonicus etwas anders, indem man hier in seinem Larvenstadium, selbst gegen das Ende der Metamorphose, noch keine Spur von Knorpelbildung in der Sklera wahrnehmen kann, die ganz bindegewebig aufgebaut ist; erst unmittelbar nach der Metamorphose (Stadium 5) tritt er als ein sehr schwach ausgebildetes Knorpelplättchen auf. Daraus kann man sagen, dass beim Bufo der Zeitpunkt des ersten Vorkommens des Skleralknorpels der Übergangszeit rom Wasser- zum Landleben sehr nahe liegt. Nachher, während des Landlebens, entwickelt sich der Knorpel verhältnismässig langsam und bekommt erst in der Zeit von ca. $31 \mathrm{~mm}$ Stammlänge eine eigentümliche Schalenform.

Die Frage, warum dieses besondere Terhältnis sich nur beim Bufo zeigt, kann ich nicht sicher beantworten; doch bin ich geneigt, anzunehmen, dass dies einen gewissen Zusammenhang mit dem Moment hat, dass beim Bufo im Vergleich mit den anderen Formen der Zeitraum vom Beginn. der Entwicklung bis zum Ende der Metamorphose, wobei der ganze Körper wohl bedeutend kleiner ist als bei den anderen Arten, viel kürzer dauert und das Auftreten des Knorpels auf das spätere viel längere Landleben sich verschiebt.

Iras die Form des Skleralknorpels bei den Auuren anbetrifft, so finclet man immer eine schalenförmige Einlagerung von verschiedener 
Ausdehnung vor. Nach Bronn ('73-78) und Carrière ('85) besitzen alle Batrachier eine ,, Knorpelkapsel.“

Nach Helfreich ('70) ist der Skleralknorpel am hinteren Pol des Bulbus beim Frosch am stärksten und verdünnt sich rasch nach rorn, um kurz vor der Insertionszone der geraden Augenmuskeln mit einem abgerundeten Rande zu enden, dagegen zeigt die den äusseren Teil der Sklera bildende Bindegewebslage bezüglich ihrer an den verschiedenen Stellen rerschiedenen Dimension ein gerade umgekehrtes Verhalten. Die Angaben von Gaupp ('04) decken sich mit denen von Helfreich, und er schildert in seiner "Anatomie des Frosches" ziemlich genau den Skleralknorpel; im folgenden zitiere ich einige Zeilen daraus: „Der Knorpel der Sklera wird am Augenhintergrunde (temporal von dem proximalen Pol) durch den Sehnerven durchbohrt. Das dadurch gebildete Foramen opticum sklerae begrenzt er mit einem verdünnten zugeschärften Rand, doch verdickt er sich rasch auf das Maximum seiner Dicke, um sich distalwärts wieder zu verdünnen und vor der Insertionszone der geraden Augenmuskeln ganz aufzuhören. In der Umgebung des Sehnerven wird er von einigen kleinen Löchern für die Nn. und Aa. ciliares durchbohrt. Er besteht aus einer wenige Zellagen dicken Schicht hyalinen Knorpels ohne weitere Besonderheiten."

Ebenso auch die von mir untersuchten ausgewachsenen Anuren tragen immer eine Knorpelschale von wechselnder Mächtigkeit je nach den Arten der Tiere. Als Ganzes bildet der Skleralknorpel eine eiförmige Schale, deren dicke, erweiterte Fläche nach temporal und die dünne Spitze nach nasal zu gerichtet steht. Die Dicke der Knorpelschale ist überall nicht glejchmässig, sondern an der Temporalwand und in der Umgebung des Foramen opticum sclerae am dicksten und nimmt auch distalwärts langsam ab, um hier mit einem spitzigen Rand zu endigen. Besonders ist die Nasalwand am dünnsten und die Tentralwand etwas dicker als die dorsale. Distalwïrts reicht der Knorpel bis vorn von der Ansatzzone der geraden Augenmuskeln und endigt hier mit scharfem Rand, wobei er nicht eine wie geschnittene Ebene, sondern je nach den Stellen eine etwas unregelmässige Ebene zeigt. Das Foramen opticum sclerae stellt ein rundliches Loch dar und ist mit einem rasch verdünnten zugeschärften Rand begrenzt, doch verdickt sich der Knorpel kurz peripher von diesem Rand besonders stark; diese Verdickung bildet auch einen Ring um das betreffende Foramen.

Was die Lage des Foramen opticum anbetrifft, so liegt er nicht im proximalen Pol der Knorpelschale, sondern steht etwas weiter temporalwärts verschoben. In der nächsten Nähe des Foramen konnte ich in 
keinem Falle kleine Löcher vorfinden, im Gegensatz zu Gaupps Befund bei Anuren und meinem beim Megalobatrachus, wo die Gefässlöcher in nächster Nähe des Foramen opticum liegen. Einige Gefässlöcher waren etwas davon entfernt in der Nähe der Ansatzzone der geraden Augenmuskeln in der Dorsal- und Ventralwand der Knorpelschale zu sehen.

Ich möchte hier den Modus der Ausbildung des Skleralknorpels bei den Anuren etwas besprechen. Wie mehrmals beschrieben, bietet der Zeitpunkt des ersten Auftretens knorpeliger Elemente in der Sklera je nach den Arten ein etwas abweichendes Verhalten dar. Jedoch, abgesehen vom Bufo, beginnt in den meisten Fällen die Knorpelbildung in einem verhältnismässig frühen Larvenstadium, wo das Chondrokranium schon ziemlich gut entwickelt ist, und zwar die Ohrkapseln fast vollständig verknorpelt sind. Die Stelle, wo der Knorpel zuerst zum Vorschein kommt, ist der temporoventrale Teil des Augapfels in der Nähe oder entsprechend der Ansatzzone der Augenmuskeln. Da bei Rana, Hyla, Rhacophorus und Polypedates die nachherige Knorpelbildung in kurzem Zeitraum fast gleichzeitig in allen Wänden des Bulbus stattfindet, besonders bei beiden letzteren am bedeutendsten, so konnte ich auf Schnittpräparaten den Modus und Verlauf der Knorpelbildung nicht mit Genauigkeit verfolgen. Beim Bufo herrscht das entgegengesetzte Verhalten, sodass die Knorpelbildung verhältnismässig allmählich erfolgt, der Knorpel in der faserigen Sklera jedoch viel deutlicher ausgeprägt scharf begrenzt ist. Ich konnte mit Hilfe der Wachsplattenmodelle die Entwicklungsweise genauer beobachten. Auch hier tritt der Knorpel zuerst im temporoventralen Abschnitt des Augenhintergrundes auf. Danach erscheinen die knorpeligen Elemente nacheinander auch an den dorsalen und nasalen Abschnitten des Augenhintergrundes ohne Zusammenhang mit dem temporoventral eher ausgebildeten Knorpel, während der letztere bald durch weitere Verbreiterung mit den neugebildeten sich verbindend eine grosse Knorpelplatte von unregelmässiger Form darzustellen kommt. So sieht man jetzt den Skleralknorpel in einer ringförmigen Anordnung im Augenhintergrund, wie es bei gewisser Entwickelungsstufe von Hynobius nebulosus und Onychodactylus japonicus zu konstatieren ist. Endlich wird aus diesem Knorpelring eine eigentümliche Knorpelschale ausgebildet. Im Vergleich mit den auderen Wänden wird die Nasalwand der Schale in viel späterem Stadium vervollständigt. Während der Skleralknorpel als eine fast vollständige Schale auftritt, kann man an der distalen Nasalwand noch einen Knorpelbildungsvorgang wahrnehmen. Ein ähnlicher Befund ist schon von C. Kohl ('92) beim Proteus 
(zitiert bei Stadtmüller ('14)) geschildert worden. Nach diesem Autor wird die Sklera bei Proteus aus ungemein fest geschichteten Bindegewebsfasern gebildet, zwischen denen sich schon bei ziemlich kleinen Olmen in der hinteren Hälfte der Sklera Einlagerungen von einzelnen Knorpelzellen und Knorpelplättchen finden; diese knorpeligen Elemente mehren sich bei zunehmendem Wachstum und bilden schliesslich im hinteren Drittel der Haut um das Auge einen geschlossenen Ring von sehr schwankender Breite. Bei einem sehr grossen Proteus hatte diese Verknorpelung einen so hohen Grad erreicht, dass fast die ganze hintere Hälfte der Sklera, bis nahe gegen den Äquator des Bulbus hin, einen Knorpelbecher einschloss, der im proximalen Augenpol nur eine öffnung für den Durchtritt des Sehnerven besass. Leider konnte ich aber bei diesem Autor keine Angaben bezüglich der genaueren Verhältnisse der Knorpelsubstanzen, welche nacheinander in der Sklera vorkommen, zum Bulbus finden, und die Zusammenhänge zwischen den vorher ausgel,ildeten und den nachher aufgetretenen Knorpelelementen. Aber auf Grund der etwas miteinander übereinstimmenden Verhältnisse zwischen meinem Befunde und Kohls, scheint es mir zweifellos zu sein, dass der Skleralknorpel wenigstens bejm Bufo vulgaris japonicus und Proteus im Entwickelungsverlauf einen fast gleichen Torgang durchmacht.

Der Tentral-, Temporal- und Nasalrand des Foramen opticum sclerae werden zuerst ron der temporoventral aufgetretenen Knorpelsubstanz umschlossen, während die Dorsalwand durch die Vereinigung mit der eben beschricbenen Knorpelsubstanz und einem anderen neugebildeten Knorpelstückchen, das in der dorsalen Wand und zwar in der Nähe der Eintrittstelle des Opticus auftritt, zusammengeschlossen zu sein scheint.

Es ist besonders herrorzuheben, dass der Skleralknorpel von ausgewachsenen Hynobius und Onychodactylus noch eine ringförmige Anordnung bewahrend cin ganz gleiches Terhalten wie eine gewisse Entwickelungsstufe ron dem des Bufo (Stadium 7) zeigt, wenngleich er in einer ganz entgegengesetzten Entwickelungsrichtung, d.h. bei den ersteren in einem Rückbildungs- und beim letzteren in einem Ausbildungszustand steht. Beim Diemictylus (Yano('26)) tritt die Rückbildung und das Terschwinden des Skleralknorpels zuerst im nasalen Abschnitt des Bulbus auf, wo er bei den Anuren am spätesten gebildet wird, danach folgt in der Tentral- und Dorsalwand hie und da die Unterbrechung. Die Dorsalwand zeigt die Neigung zur Rückbildung verhältnismässig früher als die Tentralwand und der Knorpel geht endlich entweder vollständig zu grunde oder bleibt selten in Form ron 
nur klcinen Stückchen im Temporoventralabschnitt des Augapfels zurück, wo er bei den Anuren zuerst rorkommt.

In Bezug auf die Herkunft des Skleralknorpels herrschen heutzutage zweierlei, entgegengesetzte Ansichten. Es ist wohl bekannt, dass bei vielen Selachiern der Skleralknorpel in der Nïhe der Eintrittstelle des Sehnerven eine gelenkartige Fläche bietet, die mit einem rom Kranium ausgehenden Knorpelfortsatz (Bulbusstütze), durch lockeres Bindegewebe angeschlossen, artikuliert. Der Fortsatz erscheint terminal sehr verschieden, bald in eine scheibenförmige oder eine quadratische Platte übergehend, bald in Knopfform endigend. Trom Kranium geht er stets von der gleichen Stelle aus, an der Orbitalwand, hinter dem Foramen nervi optici (Gegenbaur). Gegenbaur dürfte hierin mit Recht einen altererbten Zustand, einen Hinweis darauf, dass auch der Skleralknorpel selbst rom Kranium deriviert ist, erblicken (Franz). Ga u p p ('06) sagt: „Ich habe gezeigt (Anatomie des Frosches, Bd. 3, 1904, p. 772), dass wenigstens beim Frosch die Vagina fibrosa des Sehnerven und damit auch die Sklera des Bulbus nicht als eine Dural-, sondern als eine Kranialscheide aufzufassen ist, dass somit der Skleralknorpel auf eine Stufe mit dem Knorpel des Primordialkraniums gestellt werden kann. Damn aber wäre doch zu erwägen: Ist nicht vielleicht der Skleralknorpel als ein Teil des Primordialkraniums aufzufassen, der sich erst sekundär, unter dem Einfluss der Muskeln abgliederte? Bedenkt man, dass das Auge nur ein peripher vorgeschobener Gehirnteil ist, so ist eine solche Vorstellung nicht nur nicht absurd, sondern im Gegenteil sehr naheliegend. Dann aber wäre gerade der Hinweis auf den Skleralknorpel am allerwenigsten geeignet, die Auffassung ron der spezifischen Sinneskapselnatur der Nasen- und Ohrkapsel zu stützen."

Schaffer ('07) betont, dass der Epiglottis-Knorpel, gewisse Sesamknorpel, Herzknorpel, Skleralknorpel, Lidknorpel u.s.w. keine primitive, sondern eine sekundäre Verknorpelung sind.

Nach Tsusaki ('22): „Bei Larven ron Diemictylus, wo die Trabekel und Ohrkapsel noch nicht differenziert sind, entwickelt sich der Skleralknorpel gar nicht, während man erst bei der vollstïndigen Differenzierung der ersteren einen solchen deutlich wahrnehmen kamn. Auf weiterer Entwickelungsstufe berührt der Knorpelring vorn die Nasenkapsel, befindet sich aber hinten von der Ohrkapsel etwas entfernt. Der Knorpelring ist vom Kranialknorpel gut isoliert und man kann keinen Zusammenhang zwischen beiden Knorpelteilen finden." Bei vielen Anuren kommt der Skleralknorpel zuerst im Anfangstadium der Metamorphose nur als eine kleine Knorpeleinlagerung vor, wo das 
Primordialkranium jedoch bereits eine ziomlich gute Ausbildung aufweist. Besonders beim Bufo konnte ich selbst am Tiere gegen das Ende der Metamorphose keine spur von Knorpel wahrnehmen, die Sklera war ganz bindegewebig aufgebaut, wïhrend das Chondrokranium hierbei schon ganz genügende Ausbildung erfahren hat. Dagegen kommt erst am Tiere gleich nach der Metamorphose eine sehr schwache Knorpeleinlagerung vor. Und im Anfangstadium der Knorpelentwickelung treten die neugebildeten Knorpelelemente hie und da zerstreut in der Nähe der Ansatzzone der Augenmuskeln auf, ohne Zusammenhang mit der vorher ausgebildeten Knorpelsubstanz. Ausserdem hat neuerdings Nagamatsu ('24) geäussert, dass bei dem Chondrokranium von Kaulquappen rlie Vorknorpelgrundsubstanz aus den noch nicht ganz differenzierten Mesenchymzellen gebildet wird, währenrl sie bei dem Skleralknorpel aus dem Fasergewebe gebildet wird. Tach dem bisher -Erwähnten wäre es vielleicht kein Irrtum anzunehmen, dass dem Skleralknorpel nicht eine primordiale, sondern eine sekundäre Natur zuzuschreiben ist.

Schliesslich möchte ich etwas über die funktionelle Bedeutung des Skleralknorpels sagen. Bei vielen Urodelen verschwindet der Skleralknorpel beim Übergang rom Wasser- zum Landleben, während im wasserlebigen Larvenstadium fast immer ein sehr gut ausgebildeter Knorpelring rorkommt. Wenigstens bei solchen Fällen scheint mir das Vorkommen und Terschwinden des Skleralknorpels ohme Zweifel mit der Lebensweise les Tieres in gewissem Zusammenhang zu stehen. Stadtmüller ('14) meint, dass die Knorpeleinlagerung in der Sklera vielleicht zur Erhöhung ihrer Festigkeit dienen könnte, und dass rielleicht auch an die Druckschwankungen beim Aufenthalt der Tiere bald in höheren, bald in tieferen Regionen des Wassers zu denken wäre und die Rückbildung der starren Knorpeleinlagerung in der Sklera möglicherweise einen Umbau des Auges in diesem Sime zustande bringen könnte. Auch wäre es denkbar, dass die Skleralknorpel dem Sehorgan durch ihre Form einen besonderen Charakter gäben, der sich bei dean Verschwinden in der Weise umwandelte, dass damn die Länge der Augenachse eine Veründerung erfahren könnte. So betont er, dass vielleicht der Augapfel sich dem Einfluss der dünneren und 'dichteren Medien, worin das Tier lebt, anpasse. Dagegen fanden Okajima und Tsusaki ('21) beim Hynobius und Onychodactylus auch bei den ausgewachsenen Tieren einen sehr schön ausgebildeten Knorpelring, wenngleich in ihm eine kurze Unterbrechung zu finden war; daraus schlossen die beiden Autoren, dass das Verschwinden des Skleralknorpels keinen 
Zusammenhang mit der Lebensweise hat, sondern irgend einen anderen Grund hat. Neuerdings hat Stadtmüller ('25) geäussert, dass bei den Fällen von Okajima und Tsusaki der Skleralknorpel der ausgewachsenen Exemplare von Hynobius und Onychodactylus als unterbrochener Ring vorkommt, während er bei den Larven einen durchaus vollkommenen Ring darstellt; diese Unterbrechung des Knorpelringes an einer oder mehreren Stellen gäbe dem Bulbus eine passive Umgestaltungsfähigkeit.

Jedoch scheint es mir, dass solche kurze Unterbrechung des Ringes der Gestalt des Auges keine bedeutende Veränderung unmittelbar bringen kann. Bei vielen Fällen, wie schon beschrieben, kommt das knorpelige Element schon im verhältnismässig frühen wasserlebigen Larvenstadium vor und gegen das Ende der Metamorphose erweist es sich als eine vollkommene Knorpelschale. Dagegen beim Bufo zeigt 'der Skleralknorpel ein ganz entgegengesetztes Verhalten, indem beim wasserlebigen Larvenstadium (wo der Bulbus viel grössere Festigkeit haben müsste als in der Luft) der Skleralknorpel noch nicht ausgebildet ist und erst in der Nähe der Übergangszeit vom Wasser- zum Landleben seine Bildung beginnt. Warum nur beim Bufo die Sache sich so verhält, kann ich nicht sicher beantworten. Aber aus obigen Befunden möchte ich annehmen, dass der Skleralknorpel wenigstens bei den Anuren durch ihre Lebensweise d.h. durch die Medien, in welchen die Tiere leben, keine Veränderungen erfahren würde, sondern dieselben zum Teil vielmehr vielleicht mit der Funktion der Augenmuskeln im innigen Zusammenhang stehen müssen, sodass beim gewaltigen Zug der Augenmuskeln, besonders des M. retractor bulbi, der Bulbus einen passenden Widerstand dafür darbieten müsste.

\section{Ergebnisse.}

Die Resultate der vorliegenden Untersuchung lassen sich folgendermassen zusammenfassen.

1. Bei den Anuren bietet die Entwickelung des Skleralknorpels je nach den Arten des Tieres ein etwas abweichendes Verhalten dar. Jedoch in den meisten Fällen (Hyla arborea japonica Günther, Rhacophorus schlegelii Günther, Polypedates buergeri Schlegel und Rana japonica Günther) beginnt seine Bildung erst in der Anfangszeit der Metamorphose und bildet er gegen das Ende derselben oder noch etwas früher eine vollkommene Knorpelschale. Beim Bufo vulgaris japonicus (Schlegel) herrscht ein ganz anderes Verhalten, 
sodass bei den wasserlebigen Larvenstadien noch keine Spur vor Knorpeleinlagerung zu sehen ist, dagegen erst gleich nach der Meta. morphose seine Bildung beginnt und er in der Zeit von ca. $31 \mathrm{~mm}$ Stammlänge eine eigentümliche Knorpelschale aufweist.

2. Die Stellen, wo die knorpeligen Elemente zuerst zum Vorschein kommen, ist die temporoventrale Wand des Augenhintergrundes in der Nähe oder entsprechend der Ansatzzone der Augenmuskeln. Da bei Hyla, Rhacophorus, Polypedates und Rana die nachherige Knorpelbildung in ganz kurzem Zeitraum fast gleichzeitig in allen Wänden des Bulbus stattfindet, so konnte ich den Modus und Verlauf der Knorpelbildung nicht genau verfolgen.

3. Im Gegensatz zu anderen Formen folgt beim Bufo die Knorpelbildung in der Sklera verhältnismässig langsam und begrenzt der Knorpel sich scharf gegen die faserige Sklera, ist also hier deutlicher markiert. Auch hier tritt der Knorpel zuerst im temporoventralen Abschnitt des Augenhintergrundes auf; danach treten die neuen Knorpelstücke an der dorsalen und nasalen Wand auf und alle diese ordnen sich im Augenhintergrund ringförmig an, um zuletzt eine eigentümliche Knorpelschale zu bilden. Die nasale Wand der Knorpelschale wird in einem viel späteren Stadium ausgebildet.

4. Als Ganzes hat die vollständig ausgebildete Knorpelschale eine eiförmige Gestalt, deren breites Ende nach temporal, das dünne etwas zugespitzte Ende nach nasal zu gerichtet steht. Die Dicke der Knorpelschale ist an der temporalen Wand und in der Umgebung des Foramen opticum sclerae am stärksten und nimmt distalwärts allmählich ab, um zuletzt kurz vor ler Insertionszone der geraden Augenmuskeln je nach den stellen in der etwas unregelmässigen Ebene aufzuhören.

5. Das Foramen opticum sclerae liegt nicht exakt im proximalen Pol der Knorpelschale, sondern steht etwas nach temporal verschoben. Es hat eine rundliche Gestalt und ist mit einem rasch zugeschärften Rand begrenzt. Der Ventral-, Temporal- und Nasalrand des Foramen sind von einer zuerst in der Temporoventralwand aufgetretenen Knorpelsubstanz direkt umgeben, während der dorsale Rand durch die sekundäre Vereinigung des eben erwähnten Knorpelstückes mit dem anderen neugebildeten dorsalen Knorpel umschlossen wird.

6. Selbst gegen Ende der Metamorphose konnte ich beim Bufo vulgaris japonicus keine Spur von Knorpelbildung in der Sklera wahrnehmen, wenngleich hier das Chondrokranium schon eine genügende Ausbildung erfahren hat; erst gleich nach der Metamorphose kommt eine sehr schwache Knorpeleinlagerung vor. Ausserdem treten im 
Anfangstadium der Knorpelentwickelung die neuen knorpeligen Elemente hie und da zerstreut in der Nähe oder entsprechend der Insertionszone der Augenmuskeln auf, ohne Zusammenhang mit der voher ausgebildeten Knorpelsubstanz. Daraus ist es mir wahrscheinlich, dass der Skleralknorpel unabhängig vom Primordialkranium, sondern erst sekundär sich entwickelt.

7. Der Skleralknorpel scheint unter einem gewissen Zusammenhang mit den Augenmuskeln entstanden zu sein, so dass er beim gewaltigen Zug der Muskeln dem Augapfel eine passende Festigkeit gibt.

Zum Schluss möchte ich Herrn Professor Dr. K. Okajima für seine freundliche Leitung und Anregung bei dieser Arbeit meinen besten Dank ausdrücken.

\section{Literaturverzeichnis.}

1. Bronn, H., Klassen und Ordnungen des Tierreiches. VI. 2. Amphibien. Leipzig und Heidelberg 1873-78.

2. Carrière, J., Sehorgane der Tiere, vergleichend anatomisch dargestellt. München und Leipzig 1885.

3. Franz, V., Sehorgan. Lehrb. d. vergl.-mikrosk. Anat. der Wirbeltiere, herausgeg. von A. Oppel. Jena 1913.

4. Gaupp, E., Anatomie des Frosches. Braunschweig 1904.

5. — Über allgemeine und spezielle Fragen aus der Lehre vom Kopfskelett der Wirbeltiere. Verhandl. d. Anat. Gesellsch. Ergänzungsheft zum XXIX. Band. d. Anat. Anz. 1906.

6. Gegenbaur, C., Vergleichende Anatomie der Wirbeltiere. Leipzig 1898.

7. Helfreich, F., Über die Nerven der Conjunctiva und Sklera. Würzburg 1870.

8. Kohl, C., Rudimentäre Wirbeltieraugen. Bibl. zool. Cassel 1892.

9. Nagamatsu, H., Morphologische Studien über die Zellen des hyalinen Knorpels. Fukuoka Ikwadaigaku Zassi. Bd. 17. 1924.

10. Okajima, K., und Tsusaki, T., Beiträge zur Morphologie des Skleralknorpels bei den Urodelen. Zeitschr. f. Anat. und Entw. Bd. 60. 1921.

11. Schaffer, J., Zur Histologie, Histogenese und phylogenetischen Bedeutung der Epiglottis. Anat. Heite. Bd. 33. 1907.

12. Stadtmüller, F., Ein Beitrag zur Kenntnis des Vorkommens und der Bedeutung hyalinknorpeliger Elemente in der Sklera der Urodelen. Anat. Hefte. Bd. 51. 1914.

13. — - Studien an Urodelenschädeln. I. Zur Entwickelungsgeschichte des Kopfskeletts der Salamandra maculosa. Zeitschr. f. Anat. und Entw. Bd. 75. 1925. 
14. Steineger, L., Herpetology of Japan and adjacent territory. Washington 1907.

15. Tsusaki, T., Zur Morphologie des Skleralknorpels von Diemictylus pyrrhogaster. Folia. anat. japon. Bd. 1. 1922.

16. Yano, K., Zur Morphologie des Skleralknorpels und -knochens bei den Uródelen. Folia. anat. japon. Bd. 4. 1926.

17. Wiedersheim, R., Vergleichende Anatomie der Wirbeltiere. Leipzig 1898.

\section{Erklärung der Abbildungen auf Tafeln XXIV.XXV.}

Fig. 1-5 sind nach den Photogrammen gezeichnet:

Fig. 1-3. Vom Wachsplattenmodelle jedes Augapfels ist nur die hintere Hälfte dargestellt. Die Knorpelsubstanz ist weisslich gezeichnet.

Fig. 4-5. Modelle des Skleralknorpels allein.

Fig. 6-10. Mikrophotogramme der Schnitte der Augäpfel.

p.n.o. Papilla nervi optici, n.o. Nervus opticus. s.k. Skleralknorpel, a.m. Augenmuskel.

Fig. 1. Modell des rechten Auges von $11 \mathrm{~mm}$ langem Bufo vulgaris japonicus (gleich nach der Metamorphose), 66 fach vergrössert modelliert und auf ca. $1 / 2$ verkleinert gezeichnet.

Fig. 2. Modell des rechten Auges von $13 \mathrm{~mm}$ langem Bufo vulgaris japonicus, 50 fach vergrössert modelliert und auf ca. 1/2 verkleinert gezeichnet.

Fig. 3. Modell des rechten Auges von $16 \mathrm{~mm}$ langem Bufo vulgaris japonicus, 50 fach vergrössert modelliert und auf ca. $1 / 2$ verkleinert gezeichnet.

Fig. 4. Modell des Skleralknorpels des linken Auges von $27 \mathrm{~mm}$ langem Bufo vulgaris japonicus, 33 fach vergrössert modelliert und auf ca. $1 / 2$ verkleinert gezeichnet.

Fig. 5. Modell des Skleralknorpels des rechten Auges von $57 \mathrm{~mm}$ langem Bufo vulgaris japonicus, 20 fach vergrössert modelliert und auf ca. $1 / 2$ verkleinert gezeichnet.

Fig. 6. Senkrechter meridionaler Sclınitt durch linkes Auge eines $11 \mathrm{~mm}$ langen Bufo vulgaris japonicus (gleich nach der Metamorphose). Vergx. ca. $98 \mathrm{mal}$.

Fig. 7. Senkrechter meridionaler Schnitt durch rechtes Auge einer $30 \mathrm{~mm}$ langen Kaulquappe von Hyla arborea japonica. Vergr. ca. 98 mal.

Fig. 8. Senkrechter meridionaler Schnitt durch den Temporalteil des rechten Auges einer $30 \mathrm{~mm}$ langen Kaulquappe von Rhacophorus schlegelii. Vergr. ca. $98 \mathrm{mal}$.

Fig. 9. Senkrechter meridionaler Schnitt durch linkes Auge einer $33 \mathrm{~mm}$ langen Kaulquappe von Polypedates buergeri. Vergr. ca. 78 mal.

Fig. 10. Horizontaler meridionaler Schnitt durch rechtes Auge einer $36 \mathrm{~mm}$ langen Kaulquappe von Raha japonica. Vergr. ca. 98 mal. 
Fig. 1.

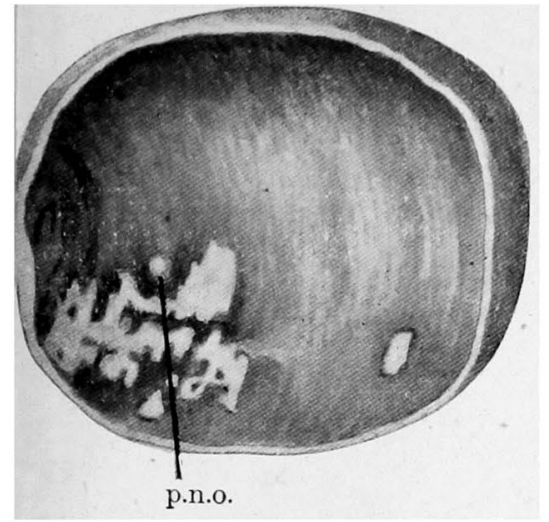

Fig. 2.

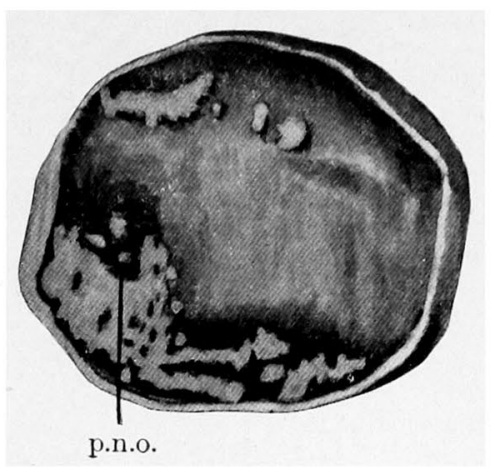

Fig. 3.

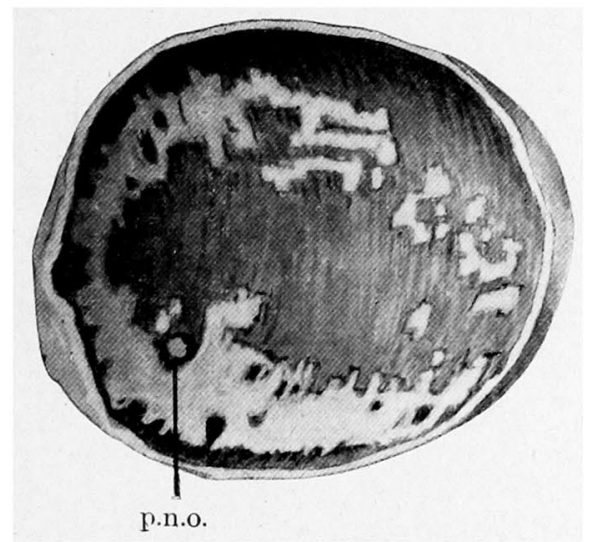

Fig. 4.

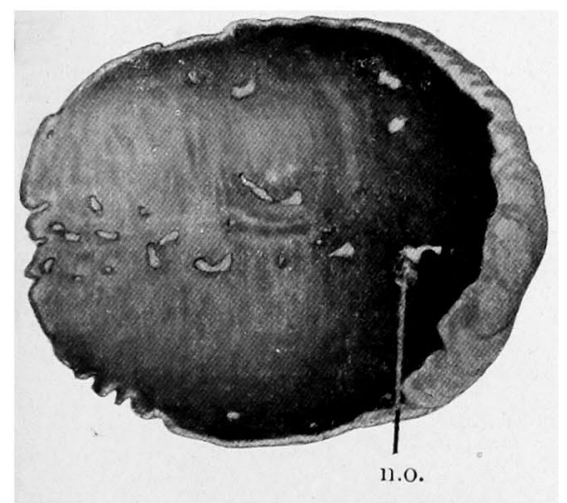

K. Yano.
Fig. 5.

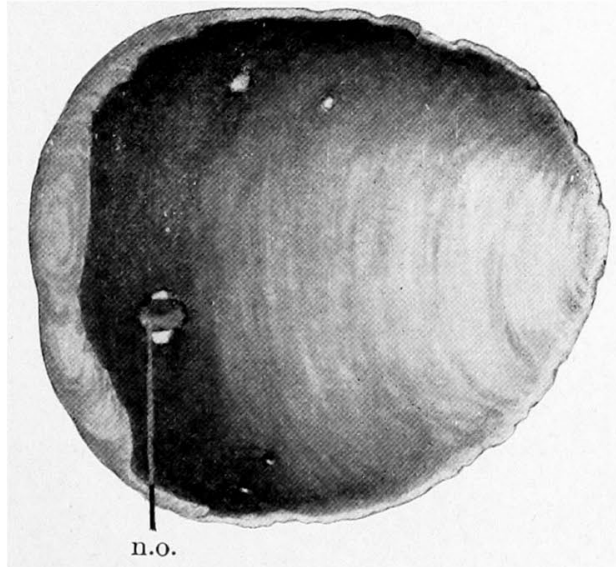


A ANATOMICA JAPONICA, BD. V.

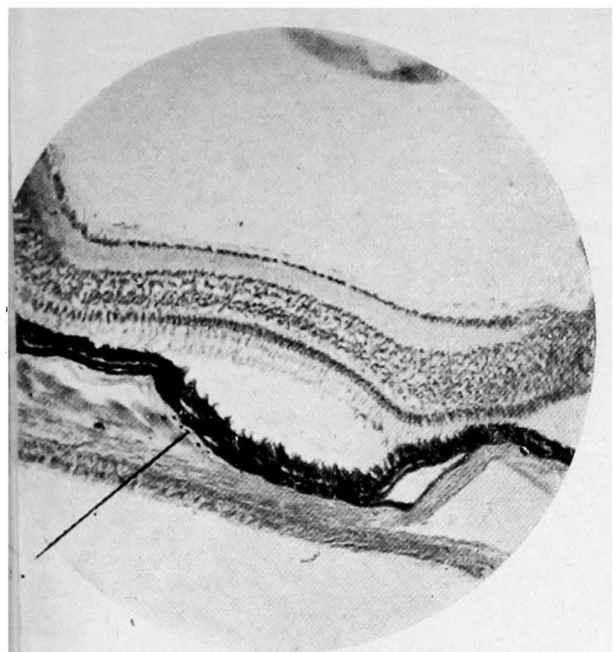

Fig. 6.

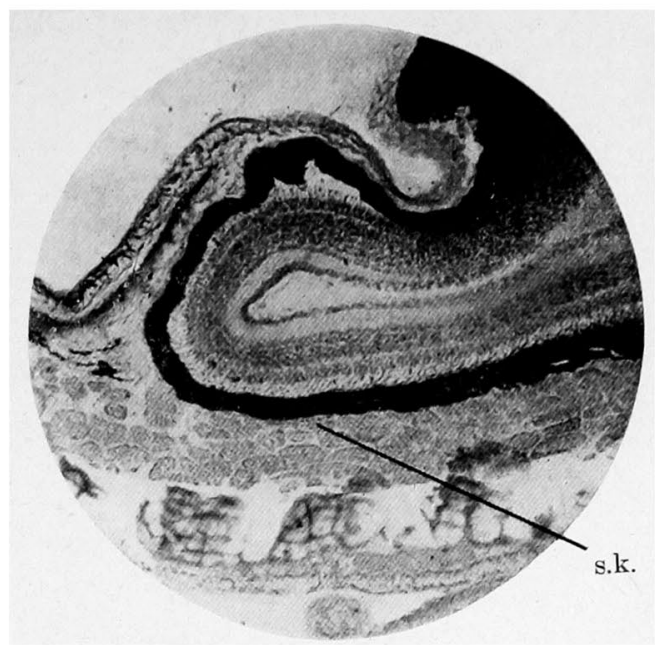

Fig. 7.

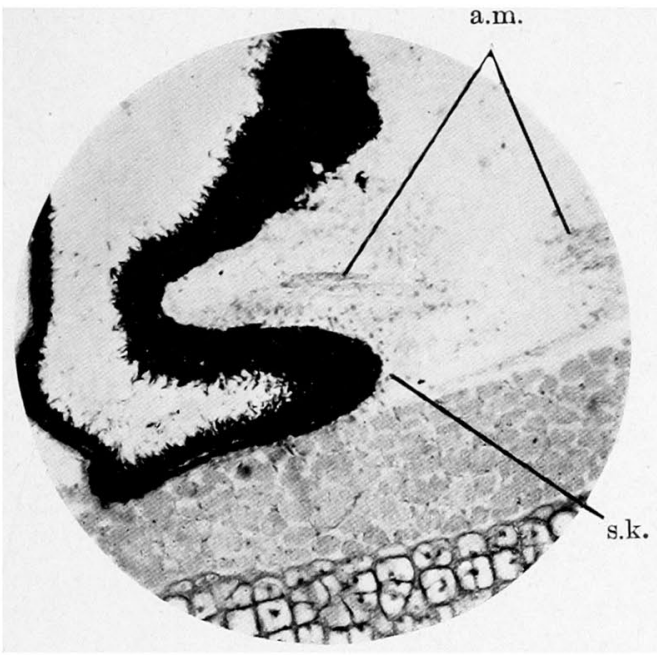

Fig. 8 .

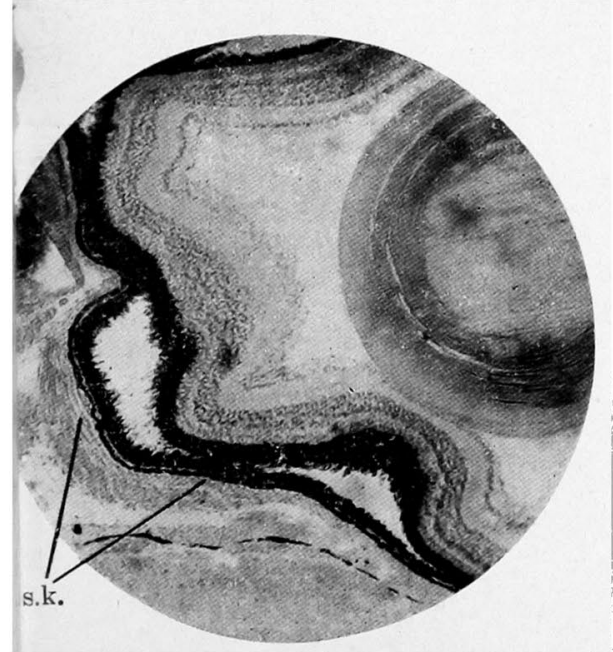

$\therefore$ Yano.

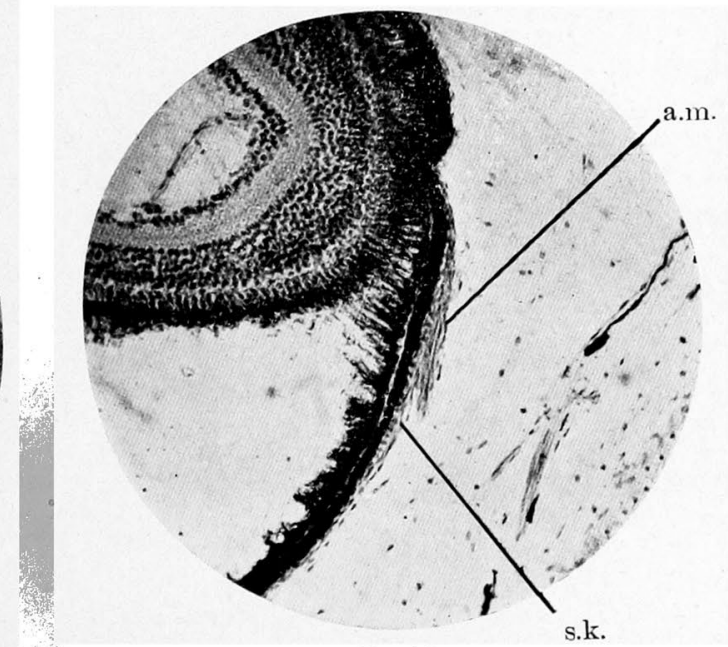

Fig. 10. 